\title{
Magnetic reconnection driven by intense lasers
}

\author{
Jiayong Zhong ${ }^{1,2}$, Xiaoxia Yuan ${ }^{1}$, Bo Han ${ }^{1}$, Wei Sun ${ }^{1}$, and Yongli Ping ${ }^{1}$ \\ ${ }^{1}$ Department of Astronomy, Beijing Normal University, Beijing 100875, China \\ ${ }^{2}$ IFSA Collaborative Innovation Center, Shanghai Jiao Tong University, Shanghai 200240, China \\ (Received 29 November 2017; revised 8 June 2018; accepted 11 June 2018)
}

\begin{abstract}
Laser-driven magnetic reconnection (LDMR) occurring with self-generated B fields has been experimentally and theoretically studied extensively, where strong B fields of more than megagauss are spontaneously generated in highpower laser-plasma interactions, which are located on the target surface and produced by non-parallel temperature and density gradients of expanding plasmas. For properties of the short-lived and strong B fields in laser plasmas, LDMR opened up a new territory in a parameter regime that has never been exploited before. Here we review the recent results of LDMR taking place in both high and low plasma beta environments. We aim to understand the basic physics processes of magnetic reconnection, such as particle accelerations, scale of the diffusion region, and guide field effects. Some applications of experimental results are also given especially for space and solar plasmas.
\end{abstract}

Keywords: high energy density physics; laser plasmas interaction; plasmas astrophysics

\section{Introduction}

Magnetic reconnection (MR) is one fundamental plasma process in which magnetic energy is converted into kinetic and thermal energy. MR has been a subject of great interest to astrophysics, space, and laboratory plasmas ${ }^{[1]}$. Solar flares are the best example of the occurrence of MR. Loop-top X-ray source and X-ray jets are often observed in the solar flare, which are considered as the most directed evidence of $\mathrm{MR}^{[2]}$. Many recent observations also confirmed the powerful solar eruptions such as coronal mass ejections, which are tightly related to MR. In the Earth's magnetosphere, MR plays an important role in interactions between the magnetic fields of the solar wind and the Earth's dipole field ${ }^{[3]}$. Since the solar wind expands exceeding magnetosonic speed, a bow shock is generated surrounding the magnetosphere. The first reconnection site, dayside reconnection, is at the frontside magnetopause, where the wind field is southward or has a southward component. The second one is located at the distant magnetotail, usually called nightside reconnection. Evidence of reconnection occurring in the Earth's magnetotail has been collected by several spacecrafts, which have detected the tail-ward jet of protons $^{[3]}$.

In laboratory plasma, two categories of MR experiments were driven by different devices covering different plasma

Correspondence to: J. Zhong, Department of Astronomy, Beijing Normal University, No. 19, Xinjiekouwai Street, Haidian District, Beijing 100875, China. Email: jyzhong@bnu.edu.cn parameters. The first one is the magnetically driven system. Based on the magnetic reconnection experiment (MRX), Yamada et al. ${ }^{[4]}$ identified two different shapes of neutral sheet current layers and a thin double Y-shaped diffusion region in different conditions. Egedal et al. ${ }^{[5]}$ investigated the spontaneous magnetic reconnection, obtaining the experiment data including plasma density, magnetic flux function, reconnection rate, and the current density in the versatile toroidal facility (VTF) magnetic configuration. $\mathrm{MST}^{[6]}$, TFTR $^{[7]}$, ITER ${ }^{[8]}$, NGRX ${ }^{[9]}$ also provide plasma environment for magnetically driven reconnection experiments. The typical physics parameters of those devices are, such as Bfield about hundred to kilo Gauss, and electron density ${ }^{[10]}$ up to $n_{e}=10^{13} \mathrm{~cm}^{-3}$. The second one is the flow driven system, usually using high-power lasers and Z-pinches. Yates et al. ${ }^{[11]}$ realized the occurrence of MR when an unexpected $\mathrm{X}$-ray emission was observed between two laser spots in a multi-beams experiment. As the two bubbles expanded laterally and encountered each other with oppositely directed magnetic fields, reconnection took place and the field lines were topologically rearranged in the diffusion region. Later, with the proton radiography technique, Nilson et al. ${ }^{[12]}$ and Li et al. ${ }^{[13]}$ diagnosed the laser-driven magnetic reconnection (LDMR), and some striking features were found, such as the collimated jets and magnetic null point in the diffusion region. Rosenberg et al. ${ }^{[14]}$ utilized proton probing to study asymmetric MR between two pulses arriving with a temporal delay between them. 
There are several textbooks and review papers to introduce the history of theories and observations of MR. In this paper, we take the chance that readers have the basic knowledge of MR. We give a short review for the recent LDMR experiments both in low and high beta plasmas. Ample references are also provided, so that more in depth reading can easily be pursued. In Section 2 we introduce the generation of magnetic fields in laser plasmas. Section 3 will focus on the LDMR experiments, and finally we introduce some applications of those experiments to study space science and astrophysics.

\section{Magnetic fields in the laser-plasma interaction}

\subsection{Biermann battery effect of long-pulse lasers}

In a plasma, inertial effects lead to electric currents and magnetic fields. This mechanism is known as the Biermann battery effect ${ }^{[15]}$ that can be derived by combining the generalized Ohm's law with Maxwell's equations. When an intense long-pulse (nanosecond) laser drives the thin solid target to excite the plasma, at the same time due to the role of pressure, super-hot electrons are formed on the laser radiation surface. And super-hot electron flow can be transported into the target, meanwhile, one plasma bubble is generated on the surface of the target. In addition, the mass of an electron is small and can be quickly accelerated and heated by the laser radiation pressure forming an electronic compression layer. The plasma temperature and pressure rise rapidly, and a large temperature gradient and a pressure gradient are formed in the normal direction of the back surface of the target, causing an isothermal expansion and thermal expansion of plasma along the normal direction of the back surface of the target.

Meantime, the thermal conduction within the target is much more effective than the adiabatic expansion of the electrons. Therefore the temperature gradient direction of the plasma is substantially parallel to the target surface, while the density gradient of the plasma is substantially perpendicular to the target surface. The inhomogeneity of the irradiated area will increase the inconsistency in directions of the temperature gradient and of the density gradient. During the expansion, the thermoelectromotive force is generated by the inconsistency of the temperature gradient and the density gradient, which cause the thermal current and induce the self-generated magnetic field. The self-generated magnetic fields are toroidal and quasi-steady, which are concentrated on a hemispherical shell surrounding the ablated plasma bubble. They have maximum amplitude near the edge but fall to zero at the center ${ }^{[16-18]}$. The schematic diagram is shown in Figure 1. The Biermann effect has been early observed in laser-generated plasmas ${ }^{[19]}$. The plasma has strong temperature and density gradients that are nearly perpendicular, leading to a source term for the

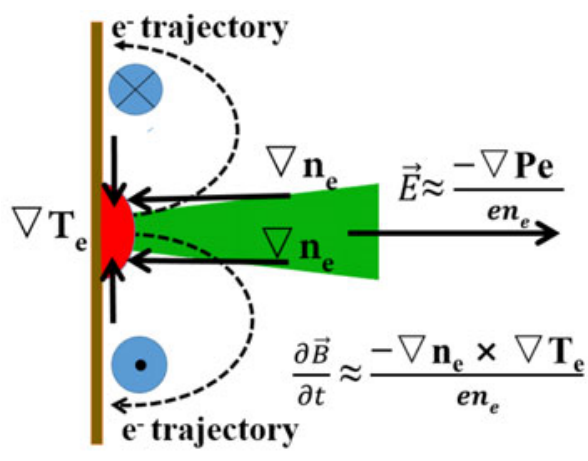

Figure 1. Schematic diagram of the annular magnetic field in the plasma.

magnetic field. These magnetic fields, typically megagauss (MG) in strength, were studied through Faraday rotation.

The self-generated magnetic field is described in the following equations. The self-generated electric field is expressed in Equation (1), Equation (2) is Faraday's law of electromagnetic induction, and Equation (3) is the equation of state. Combining these equations, we obtain Equation (4) to describe the self-generated magnetic field:

$$
\begin{gathered}
E+\frac{\nabla P_{e}}{e n_{e}}=0, \\
\frac{\partial B}{\partial t}+\nabla \times E=0, \\
P_{e}=n_{e} k T_{e}, \\
\frac{\partial B}{\partial t}=\nabla \times \frac{\nabla\left(n_{e} k T_{e}\right)}{e n_{e}}=-k \frac{\nabla n_{e} \times \nabla T_{e}}{n_{e} e},
\end{gathered}
$$

where $k$ is the Boltzmann constant, $T_{e}$ is the electron temperature, and $n_{e}$ is the electron density.

\subsection{Magnetic field produced by short-pulse lasers}

The development of the technique of chirped pulse amplification has enabled high-power lasers to produce multimegawatt femtosecond and picosecond laser pulse that can be focused on the target at relativistic intensities in the laboratory. Extraordinary strong magnetic fields are expected to develop during the interaction. These fields are predicted to exist in localized regions near the critical density surface. Such magnetic fields can be generated by several mechanisms, including: (1) by the Biermann battery effect mentioned above, (2) by the ponderomotive force associated with the laser radiation itself, and (3) by the current of fast electrons generated during the interaction. The strength of magnetic fields generated by each of these sources can reach up to hundreds of MG at high laser intensities. The ranking one is GG. All of these fields are predicted to be in the azimuthal direction around the laser interaction region.

Wagner et al. ${ }^{[20]}$ carried out experiments using the highintensity Vulcan laser system at the Rutherford Appleton Laboratory. They showed that polarization measurements 


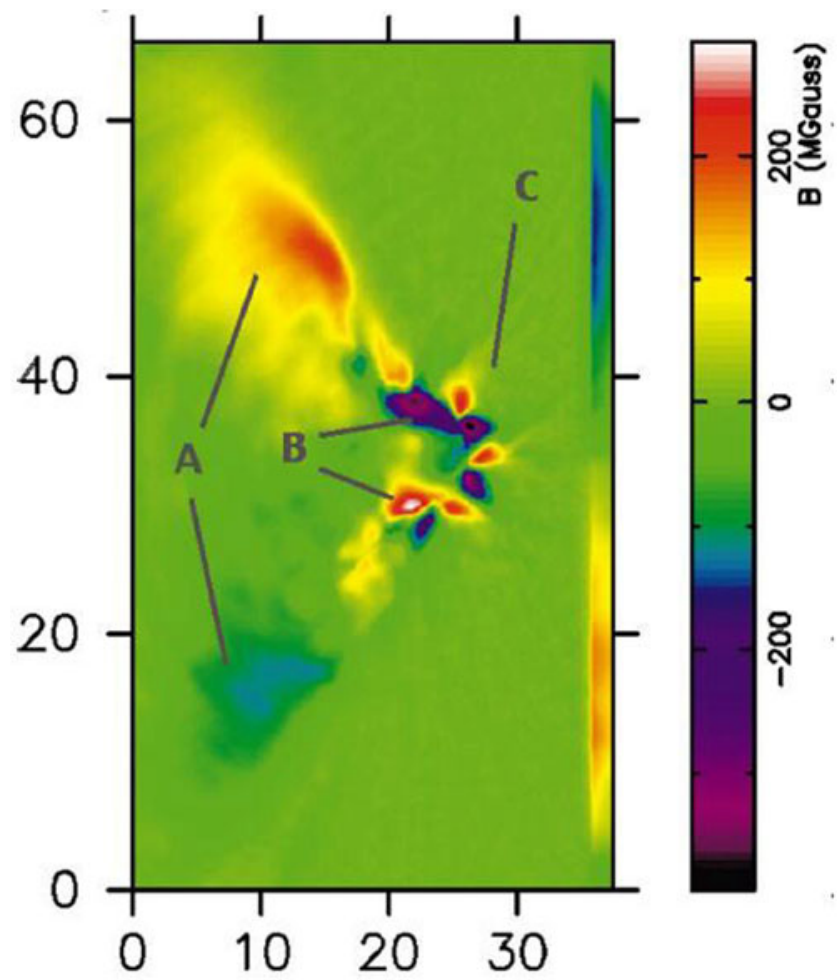

Figure 2. The simulation of 2D particle-in-cell code, OSIRIS, showing hole-boring effects and regions of magnetic field. Region A represents the non-parallel temperature and density gradients; region $\mathrm{B}$ represents the ponderomotive source; region $\mathrm{C}$ represents the magnetic fields due to Weibel-like instability from laser-generated electron beams ${ }^{[20]}$.

of high-order extreme ultraviolet (EUV) laser harmonics generated during the interaction suggested the existence of magnetic field strengths of $0.7 \pm 0.1 \mathrm{GG}$ in the overdense plasma. The polished glass targets were focused by the laser at the wavelength of $1.053 \mu \mathrm{m}$, energy up to $100 \mathrm{~J}$, laser duration of $0.7-1.2 \mathrm{ps}$, and intensity of $9 \times 10^{19} \mathrm{~W} \cdot \mathrm{cm}^{-2}$. To diagnose the strong magnetic field, a multichannel vacuum ultraviolet (VUV) polarimeter, which can probe the denser region of the plasma, has been employed. A 2D OSIRI particle-in-cell (PIC) simulation shows that there are three regions of $\mathrm{A}, \mathrm{B}, \mathrm{C}$, which represent three different magnetic field generation mechanisms. They are nonparallel temperature and density gradients, a ponderomotive source and Weibel-like instability, respectively, as shown in Figure 2.

\subsection{Magnetic fields induced by laser-coil target}

Another kind of mechanism for generating strong magnetic field is combined with the long-pulse laser and the super-hot electrons generated by the strong laser. The capacitor-coil target is composed of two disks connected by two coils, and the high-intensity long-pulse laser is focused on the center of the second disk through the hole in the middle of the

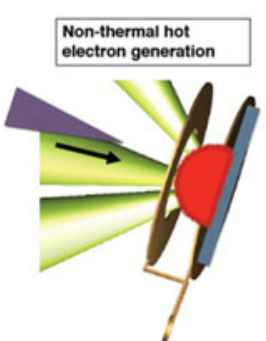

(a)

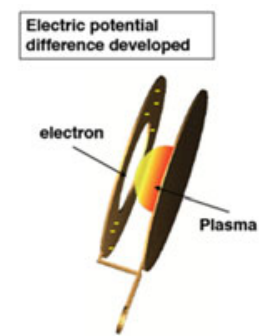

(b)

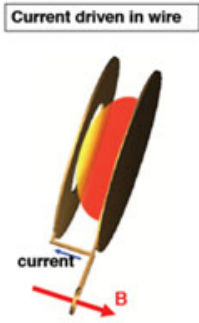

(c)
Figure 3. The capacitor-coil target. (a) Non-thermal hot electron is generated on the target surface of the capacitor coil. (b) The potential difference between the two capacitor coils is developed. (c) Loop current is generated in the coil.

first disk. The schematic diagram is shown in Figure 3(a). The plasma is generated on the center of the second disk and a large number of non-thermal electrons are emitted from the disk as well. The non-thermal electrons hit the first disk and remain on the disk, while the second disk is positively charged due to the loss of a large amount of electrons. In order to maintain the electrical neutrality, a large number of electrons on the first disk return to the second disk by the coil, and a strong current is produced in the coil. According to Faraday's law, a strong magnetic field results from the current. Law et al. conducted experiments on GEKKO-LFEX laser devices ${ }^{[21]}$. In their experiment, the two capacitor-coil targets were arranged in a geometrical shape to produce a relatively uniform magnetic field. A $50 \mu \mathrm{m}$ thick Ta plate is placed between the coil and the capacitor plate to block the plasma flow from the capacitor side, and a $250 \mu \mathrm{m}$ thick $\mathrm{CH}$ target is placed between the two coils to study magnetic field diffuse in the middle of the $\mathrm{CH}$ target. The target disk of each capacitor is driven by a GEKKO XII laser beam, where the electrons in the plasma diffuse from the target normal to the front of the target. While the plasma sheath transports from the target to the front of the target, a large difference of potential between the two coils generates a current of hundreds of ampere. Hence a strong dipole magnetic field is created. A B-field of $10 \mathrm{MG}$ has even been obtained with the laser-driven capacitor-coil target ${ }^{[22]}$.

\section{Magnetic reconnection with different plasma $\beta$}

MR is a topological restructuring of a magnetic field as a result of the change in its connectivity. Based on the understanding of magnetic fields in laser plasmas, we can construct MR with special experimental setup. In astrophysics and laboratory, complex plasma situations exist in which magnetic pressure and plasma pressure interchange the dominance. Plasma $\beta=p_{e} /\left(B^{2} / 2 \mu_{0}\right)=n_{e} k T_{e} /\left(B^{2} / 2 \mu_{0}\right)$ that is the ratio of the plasma pressure to the magnetic pressure, is used to describe the dominance force. When $\beta \gg 1$, it 


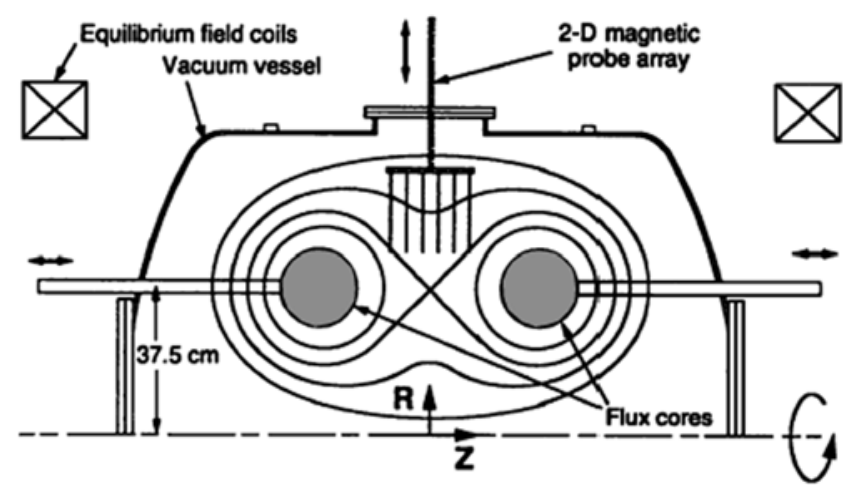

Figure 4. A schematic view of the MRX setup ${ }^{[24]}$.

means plasma pressure dominates over magnetic pressure, and when $\beta \ll 1$, magnetic pressure dominates over plasma pressure. In solar atmosphere from the photosphere to the upper corona, $\beta$ varies from $>1$ (photosphere) to $\ll 1$ (chromosphere and corona) then to $>1$ (acceleration region and solar wind) again, and $\beta$ has significant impact on the plasma dynamics ${ }^{[23]}$. In laboratory, a series of experiments have been carried out to study magnetic reconnection in low- $\beta(\beta<1)$ and high- $\beta(\beta>1)$ plasmas. Here we introduce several MR experiments in low- $\beta$ and high- $\beta$ plasma environments both in magnetic-driven and laserdriven systems.

\subsection{Magnetically driven reconnection in the low- $\beta$ plasma}

According to the key parameters of the plasma from MRX device and VTF device in references ${ }^{[24,25]}$, the plasma $\beta$ values, which are far smaller than 1 , can be calculated. A series of MR experiments were carried out on these two devices.

\subsubsection{The experiment in the MRX device}

The MRX device was built at Princeton Plasma Physics Laboratory in 1995 to investigate the fundamental physics of $\mathrm{MR}^{[24]}$. Coupling of local microscale characteristics of the reconnection layer and global properties was focused on. The experimental plasma satisfies the magnetohydrodynamics (MHD) $\left(S>10^{3}\right)$ condition, and the global plasma size is $R \sim 30-50 \mathrm{~cm}$, which is much larger than the ion gyroradius ${ }^{[26]}$. Experiments were carried out to identify the details of MR such as the shape of the diffusion region during $\mathrm{MR}^{[24]}$ and the verification of the Sweet-Parker model ${ }^{[27]}$, as well as the two-fluid effect on $\mathrm{MR}^{[4]}$.

The MRX device ${ }^{[24]}$, as shown in Figure 4, contains two flux cores, and each consists of a toroidal field (TF) coil and a poloidal field (PF) coil. Two toroidal plasmas with annular cross section are formed independently around two flux cores by pulsing programmed currents in the TF coils, after the PF coil induces a quadrupole poloidal magnetic field.

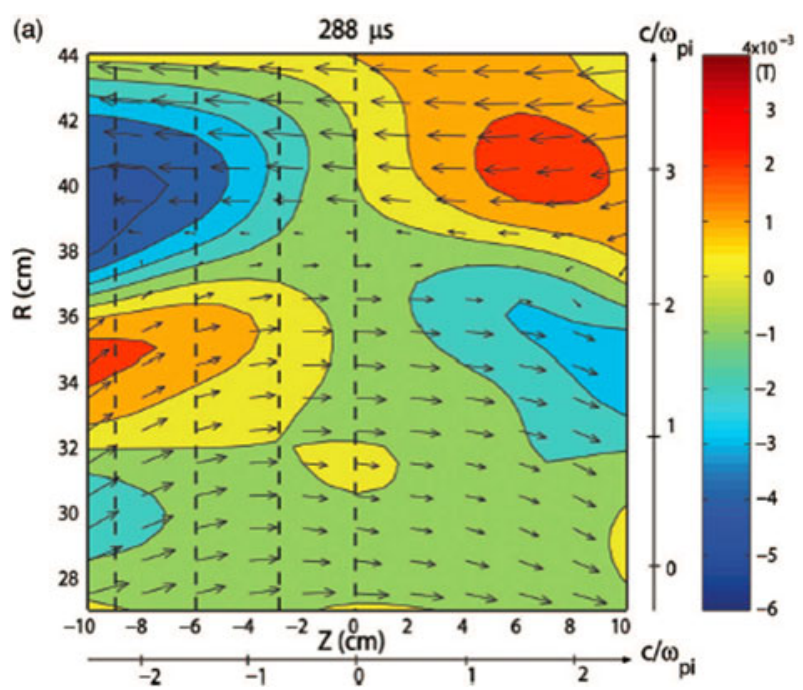

Figure 5. The contours of the out-of-plane quadrupole field in the diffusion region during reconnection ${ }^{[4]}$.

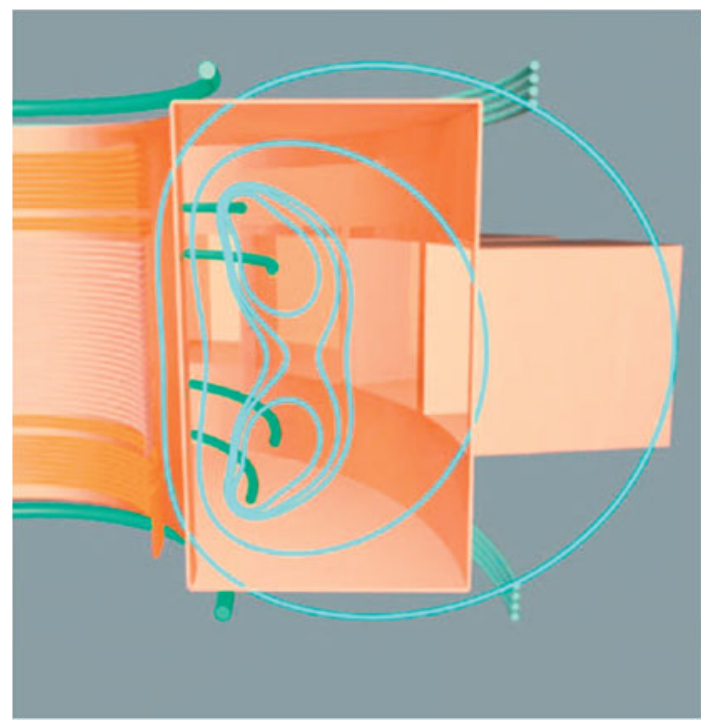

Figure 6. A schematic view of VTF experimental setup ${ }^{[28]}$.

Magnetic reconnection is driven by the programmed currents in the coils after the plasmas are induced. A 2D magnetic probe array is usually used to diagnose the magnetic field, and other diagnostics such as electrostatic probes and optical systems, are employed according to the needs. Two-fluid effects of the reconnection dynamics were investigated using the MRX device. A 90-channel probe array was scanned to measure the 2D profile of the out-of-plane quadrupole magnetic fields, and obtained the contours of the magnetic field in the diffusion region and the reconnection magnetic field vectors in the $R-Z$ plane, as shown in Figure 5. For general conditions the physical parameters are $n_{e} \sim(0.1-1)$ $\times 10^{14} \mathrm{~cm}^{-3}, T_{e}=5-15 \mathrm{eV}, B=0.2-1 \mathrm{kG}, S>500$, and the plasma $\beta$ can be calculated as $4 \times 10^{-3[4]}$. 

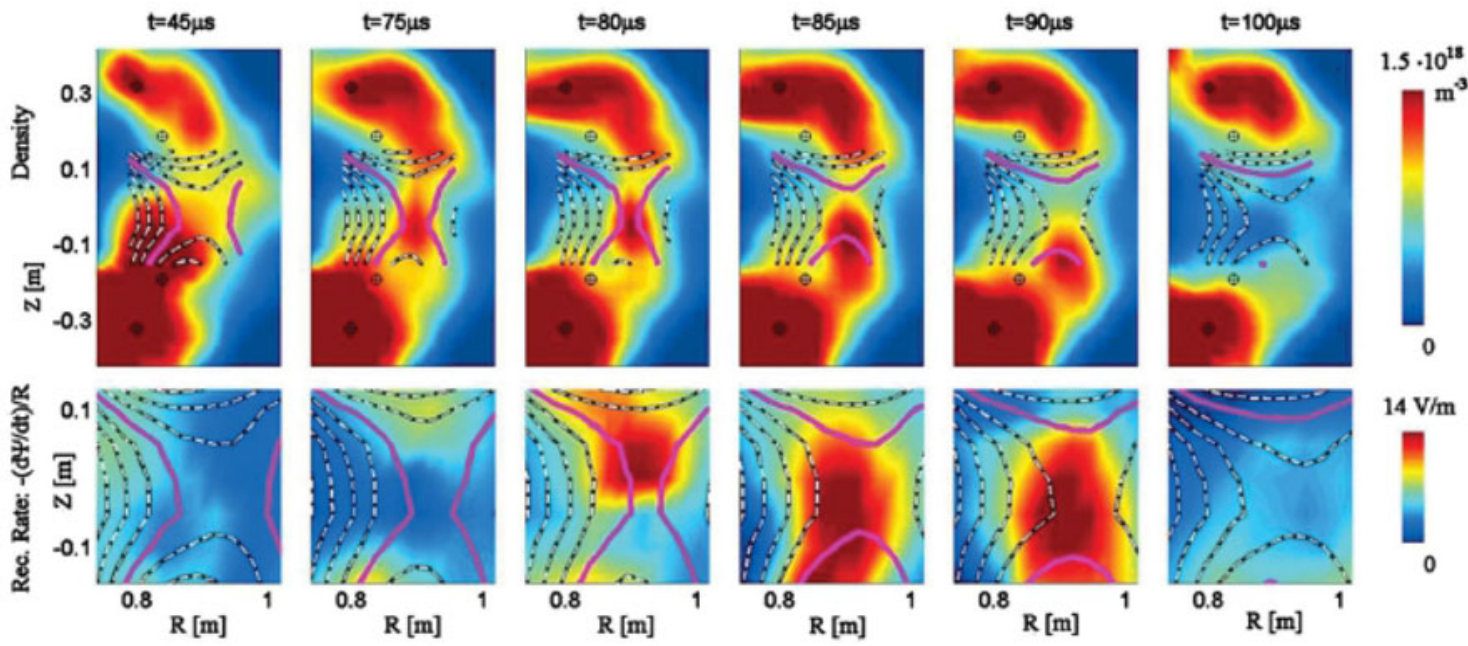

Figure 7. Measured contour of the plasma density and the reconnection rate $\mathrm{e}^{[28]}$.

\subsubsection{The experiment in the VTF}

The VTF was built at PSFC, MIT, to explore fast reconnection in collisionless plasma, where the electron mean free path is much larger than the dimensions of the plasma. And it has similar geometry with the MRX, except for the much stronger guide field ${ }^{[5]}$. Figure 6 shows the cross section of the VTF device, and the orange structures are field coils which produce a toroidal magnetic field. The blue lines show the PF geometry ${ }^{[25]}$. Thin stainless steel wires suspend four coils, causing minimal disturbance to the plasma. A guide field is produced by an external coil.

One experiment exploring the spontaneous MR with a strong guide field was performed in $\mathrm{VTF}^{[28]}$. Figure 7 shows the evolution in plasma density and reconnection rate. When $t \leqslant 75 \mu \mathrm{s}$ the reconnection rate is on the order of $2 \mathrm{~V} / \mathrm{m}$, and the current sheet is stretched to $0.1 \mathrm{~m}$. When the width of the current sheet reaches the ion-sound-Larmor radius $\rho_{s}$, the plasma is ejected at $t=80 \mu \mathrm{s}$, and the reconnection rate jumps to $14 \mathrm{~V} / \mathrm{m}$ at the upper outflow region of the current sheet, indicating that at first the reconnection process is slow, which allows the magnetic stress to accumulate in the system and a current channel to form. Other key parameters are also documented in the reference ${ }^{[28]}$. Electron heating and ions accelerated were also observed in the experiment. The key parameters of VTF, $n_{e}=1.5 \times 10^{12} \mathrm{~cm}^{-3}, T_{e}=25 \mathrm{eV}$, $B=0.044 \mathrm{~T}$, were gained, and the plasma $\beta$ can be calculated as $7.8 \times 10^{-3[29]}$.

\subsection{Laser-driven low- $\beta M R$}

3.2.1. Helmholtz coils MR with long-pulse lasers

Another way to produce a low- $\beta$ plasma environment is based on the field produced by a laser-driven Helmholtz capacitor-coil target, which supplies two antiparallel simple and explicit magnetic fields in a low density plasma as
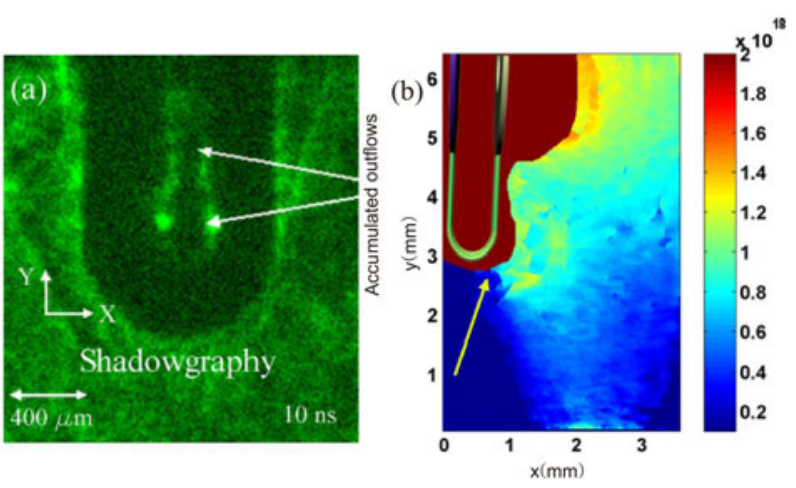

Figure 8. (a) Shadow image taken at a delay of $10 \mathrm{~ns}$. (b) The electrons distribution around the coils at a delay of $3 \mathrm{~ns}^{[35]}$.

shown in Figure 3. These targets are usually consisted of two parallel metallic foils connected by two metallic U-turn coils. Laser beams come through a laser entrance hole which is in the front foil, irradiating the rear foil. Hot electrons come out first and the front foil is charged, and an electrical potential between the two foils is built up. This results in currents in the coils which generate the antiparallel magnetic fields ${ }^{[30-34]}$. The coils are ionized by the X-ray radiation from the ablated foil and the Joule heating, producing low density plasma around the coils. And then in between the coils antiparallel magnetic fields are built where MR occurs.

Pei et al. ${ }^{[35]}$ carried out an MR experiment on GEKKO XII laser device with a Helmholtz capacitor-coil target. An accumulated plasma plume near the MR outflow was observed, as shown in Figure 8(a). The two-dimensional electron density can be obtained by Abel inverting interferogram. The electron density out of the coils is on the order of $10^{18} \mathrm{~cm}^{-3}$, as shown in Figure 8(b). Using Faraday effect, the B-field at $3.6 \mathrm{~mm}$ from the middle of the two coils can be obtained, and hereafter the B-field distribution of 


\section{OMEGA EP $K_{a}$ Images}
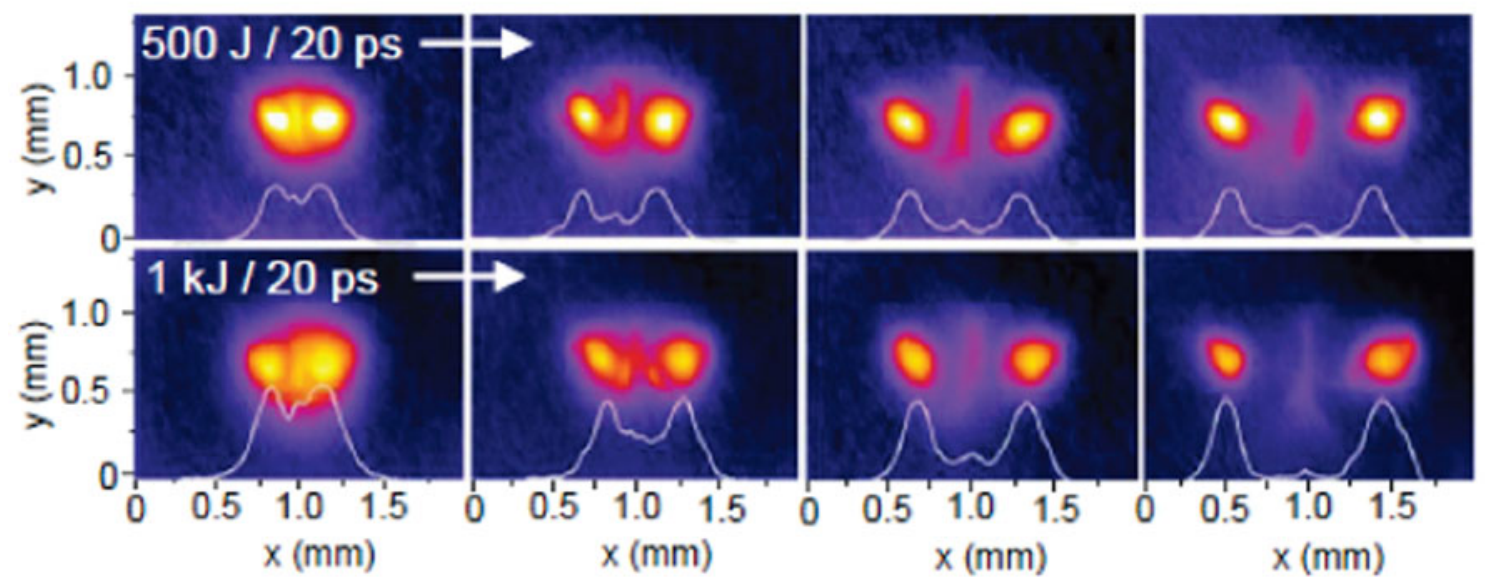

Figure 9. Frontside copper $\mathrm{K}_{\alpha}$ images from focal spot separation scans using the OMEGA EP laser. $50 \mu \mathrm{m}$ horizontal line-outs are superimposed ${ }^{[41]}$.

the coils can be simulated by Radia code ${ }^{[36]}$. The $\beta$ near the coils is $p_{e} /\left(B^{2} / 2 \mu_{0}\right)=n_{e} k T_{e} /\left(B^{2} / 2 \mu_{0}\right) \sim 0.016^{[35]}$, where the electron density is $n_{e}=10^{24} \mathrm{~m}^{-3}$, the Boltzmann constant is $k=1.38 \times 10^{-23} \mathrm{~J} / \mathrm{K}$, the electron temperature is $T_{e}=10 \mathrm{eV}=1.6 \times 10^{6} \mathrm{~K}$, and the B-field intensity is $50 \mathrm{~T}$. This is the first time using the Helmholtz capacitor-coil target to perform a low- $\beta$ MR.

\subsubsection{Low- $\beta$ MR with relativistic lasers}

In many astrophysical plasma environments, MR plays an important role in the conversion of energy ${ }^{[37]}$. For some high energy astrophysical systems, such as the brightest stellar Xray flares that emanate from the youngest stars ${ }^{[38]}$, gammaray burst ${ }^{[39]}$, and astrophysical jet from active galactic nucleus $(\mathrm{AGN})^{[40]}$, it is very difficult to directly detect the release of energy. Therefore, using the laboratory as a platform to study relativistic MR creates a breakthrough for solving the difficulties in direct measurements. Most cases of magnetic reconnection in astrophysical environments are collisionless and magnetically predominant, and system dimensions are much larger than the ion skin depth. Raymond et al. ${ }^{[41]}$ used a petawatt laser device OMEGA EP $\left(I>10^{18} \mathrm{~W} \cdot \mathrm{cm}^{-2}\right)$ to study relativistic MR. Due to the OMEGA EP laser which provides laser energy at kilojoules level with the time duration of 1 to $100 \mathrm{ps}$, relativistic electrons are generated and a strong annular self-generated magnetic field is generated by the Biermann battery effect $(\nabla n \times \nabla T)^{[42]}$ on the target surface. The plasma $\beta$ is obtained as $\beta=P /\left(B^{2} / 2 \mu_{0}\right)=$ $0.6(\tau / 2 \mathrm{ps})^{-2}(k T / 100 \mathrm{keV})-1\left(L_{T} / 25 \mu \mathrm{m}\right)^{2}\left(L_{n} / 1 \mu \mathrm{m}\right)$ $\left(n_{e} / n_{c}\right)$. For a laser of pulse duration $\tau=10 \mathrm{ps}$, a temperature of the hot electron component $k T=100 \mathrm{keV}$, a temperature gradient scale length $L_{T}=50 \mu \mathrm{m}$, a density gradient scale length $L_{n}=10 \mu \mathrm{m}$, and an electron density $n_{e}=10^{19} \mathrm{~cm}^{-3}=10^{-2} n_{c}$, we find $\beta=0.1$. Figure 9 ${ }^{[41]}$ shows the experimental results. It is found that there is locally enhanced $\mathrm{K}_{\alpha}$ emission in the midplane region. Hence, relativistic magnetic reconnection will occur between two toroidal magnetic fields, each generated by an independent laser.

Whether relativistic MR occurs is known by analyzing the X-ray emission mode, the change of the electron energy spectrum, and the time of reconnection. The energy spectrum of the accelerated particles in the midplane region is one evidence of the existence of the relativistic MR. However, there exists one risk of how to distinguish the high energy particles coming from MR or from laser acceleration itself.

The MR process occurring when the intense femtosecond laser pulse interacts with plasmas in 3D PIC simulation is reported by Ping et al. ${ }^{[43]}$. They report a relativistic PIC simulation for a three-dimensional MR process occurring in intense femtosecond laser pulse produced plasmas with a near-critical density. The two femtosecond laser pulses, parallel to each other, are shot onto a target of dense plasma layer. Fast reconnection of the GG level magnetic field inside the target is then demonstrated for the first time. In this simulation, it was shown as follows that the magnetic field expansion in space makes it possible for fast MR to take place within sub-picoseconds.

The azimuthal magnetic fields generated by two lasers from $25 T_{0}$ to $70 T_{0}$ at $z=10.7 \mu \mathrm{m}$ are shown in Figures $10(\mathrm{~g})-10(\mathrm{i})$, where $T_{0}$ is the laser period. As a reference, the field generated by a single laser pulse is also plotted in Figures 10 (a)-10(c). Clearly the azimuthal magnetic field expands laterally because of the force acting on the vortex in the $\nabla n \times \Omega$ direction ${ }^{[44,45]}$ with its topological structure 


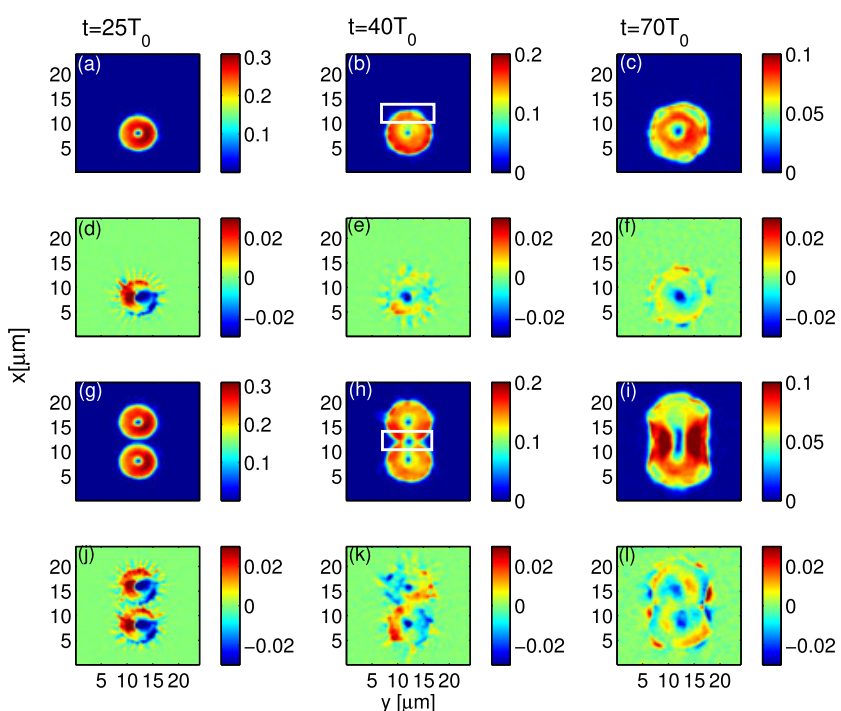

Figure 10. Snapshots (at $z=10.7 \mu \mathrm{m}$ from $25 T_{0}$ to $80 T_{0}$ ) of magnetic fields $B$. (a)-(c) Azimuthal magnetic fields $B_{\theta}$ and (d)-(f) out-of-plane magnetic fields $B_{z}$ produced by a single incident laser. (g)-(i) $B_{\theta}$ and (j)(l) $B_{z}$ by two incident lasers ${ }^{[43]}$.

kept unchanged, as an expanding bubble with an O-pointtype null at the center. At $t=40 T_{0}$ as evidently shown in Figure 10(h), the X-point-type magnetic null is then found between the two bubbles. MR occurs at this place, where the magnetic field vanishes as the magnetic bubbles with different polarities meet one another. As the process progresses, the two bubbles start to merge together into a single big bubble as shown in Figure 10(i). At $t=70 T_{0}$, the centroids of the two bubbles merge with each other completely and the new magnetic topological structure is formed. The out-of-plane magnetic fields $B_{z}$ generated by a single laser pulse and two lasers are also shown in Figures 10(d)-10(f) and Figures 10(j)-10(1), respectively. In the case of one laser (Figures 10(d)-10(f)), it is found that the magnetic field structure is always nearly centrosymmetry. In the case of two lasers (Figures 10(j)-10(1)) however, a quadrupole structure appears around the reconnection region, which is a typical signature of the Hall effect ${ }^{[25,46]}$.

Figures 11(a)-11(d) show the reconnection electric field at two characteristic moments $\left(35 T_{0}\right.$ and $\left.50 T_{0}\right)$, which are presented in Figures 10(g) and 10(h). The reconnection process is clearly collisionless since the width of the diffusion region $(1 \mu \mathrm{m}$ as shown in Figure 11(d)) is much less than the ion skin depth. The ion skin depth is calculated as $d_{i}=c / \omega_{p i} \approx$ $8.3 \mu \mathrm{m}$ at $z=10.7 \mu \mathrm{m}$, where $\omega_{p i}=\left(4 \pi e^{2} n_{0} / m_{i}\right)^{1 / 2}$ is the ion plasma frequency. However, the electron skin depth is $d_{e}=c / \omega_{p e} \approx 0.71 \mu \mathrm{m}$, where the electron plasma frequency is $\omega_{p e}=\left(4 \pi e^{2} n_{0} / \gamma_{0} m_{e}\right)^{1 / 2}$ with the relativistic factor $\gamma_{0} \approx \sqrt{1+a_{0}^{2}}$. Clearly, the reconnection region size shown in Figure 10(h) is less than $d_{i}$, then the Hall MHD model may not be applied and the electron MHD (EMHD)
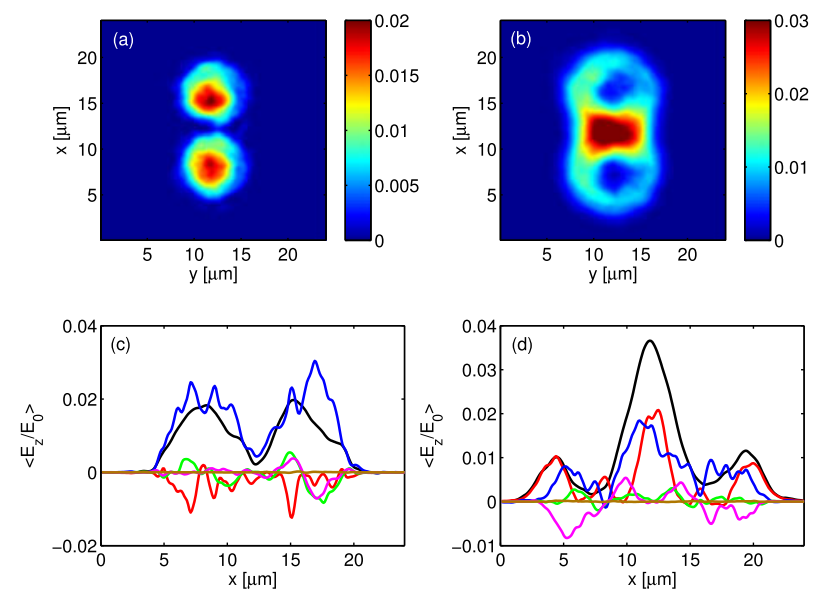

Figure 11. Reconnection electric field $E_{z}$ (at $z=10.7 \mu \mathrm{m}$ ) (a), (c) at $t=25 T_{0}$, and (b), (d) at $t=40 T_{0}$. Contributions to the generalized Ohm's law from Equation (5) along the $x$-axis at $40 T_{0}$ for $y=12 \mu \mathrm{m}$, where $\frac{1}{e\left\langle n_{e}\right\rangle}(\langle\mathbf{j}\rangle \times\langle i \mathbf{B}\rangle)_{z}$ (green line), $-\frac{1}{e\left\langle n_{e}\right\rangle}\left\langle\nabla \cdot \mathbf{P}_{e}\right\rangle_{z}$ (blue line), $\frac{m_{e}}{e^{3}\left\langle n_{e}\right\rangle}(\langle\mathbf{j}\rangle$ $\nabla\left\langle\frac{j_{z}}{n_{e}}\right\rangle$ ) (brown line), $-\frac{1}{\left\langle n_{e}\right\rangle}\left\langle\delta n_{e} \delta E_{z}\right\rangle$ (red line), and $\frac{1}{e\left\langle n_{e}\right\rangle}\langle\delta \mathbf{j} \times \delta \mathbf{B}\rangle_{z}$ (purple line $)^{[43]}$.

model should be used in the fluid approach ${ }^{[47]}$. At $t=40 T_{0}$ in this simulation, the asymptotic plasma $\beta$ is less than unity, with $\beta \sim 0.2-0.3$, even the maximum $\beta$ at the $\mathrm{X}$-point is only $\beta \sim 2.5$.

Therefore, the generalized Ohm's law of the mean reconnection electric field can be written as

$$
\begin{aligned}
\left\langle E_{z}\right\rangle= & \frac{1}{e\left\langle n_{e}\right\rangle}(\langle\mathbf{j}\rangle \times\langle\mathbf{B}\rangle)_{z}+\frac{-1}{e\left\langle n_{e}\right\rangle}\left\langle\nabla \cdot \mathbf{P}_{\mathbf{e}}\right\rangle_{z} \\
& +\frac{m_{e}}{e^{3}\left\langle n_{e}\right\rangle}\left(\langle\mathbf{j}\rangle \cdot \nabla\left\langle\frac{\mathbf{j}_{\mathbf{z}}}{\mathbf{n}_{\mathbf{e}}}\right\rangle\right)+\frac{-1}{\left\langle n_{e}\right\rangle}\left\langle\delta n_{e} \delta E_{z}\right\rangle \\
& +\frac{1}{e\left\langle n_{e}\right\rangle}\langle\delta \mathbf{j} \times \delta \mathbf{B}\rangle_{z},
\end{aligned}
$$

where the mean field of a variable $A,\langle A\rangle=\left(1 / L_{z}\right) \times$ $\int_{0}^{L_{z}} A \mathrm{~d} z$, and its fluctuation $\delta A=A-\langle A\rangle$.

In Equation (5), $\frac{-1}{\left\langle n_{e}\right\rangle}\left\langle\delta n_{e} \delta E_{z}\right\rangle$ is the electrostatic turbulence contribution and $\frac{1}{e\left\langle n_{e}\right\rangle}\langle\delta \mathbf{j} \times \delta \mathbf{B}\rangle_{z}$ is the electromagnetic (EM) turbulence contribution to the reconnection field, while the first term is the Hall field, the second is the pressure gradient, and the third is the electron inertial term. In Figures 11(c) and 11(d), the distributions of each of those terms and the reconnection electric field along the $x$-direction are given at $t=25 T_{0}$ and $t=40 T_{0}$, respectively. It can be seen that both the electron pressure tensor gradient and electrostatic turbulence dominate the reconnection process near the X-point at $t=40 T_{0}$, different from previous studies in three-dimensional reconnection where the EM turbulence plays an important role at the $\mathrm{X}$-point, while the electrostatic turbulence contribution is very small ${ }^{[48,49]}$. This is because the intensive electrostatic fluctuations propagating in the $z$ direction, which were generated by the ultra-intense laser, are injected continuously into the plasma. 


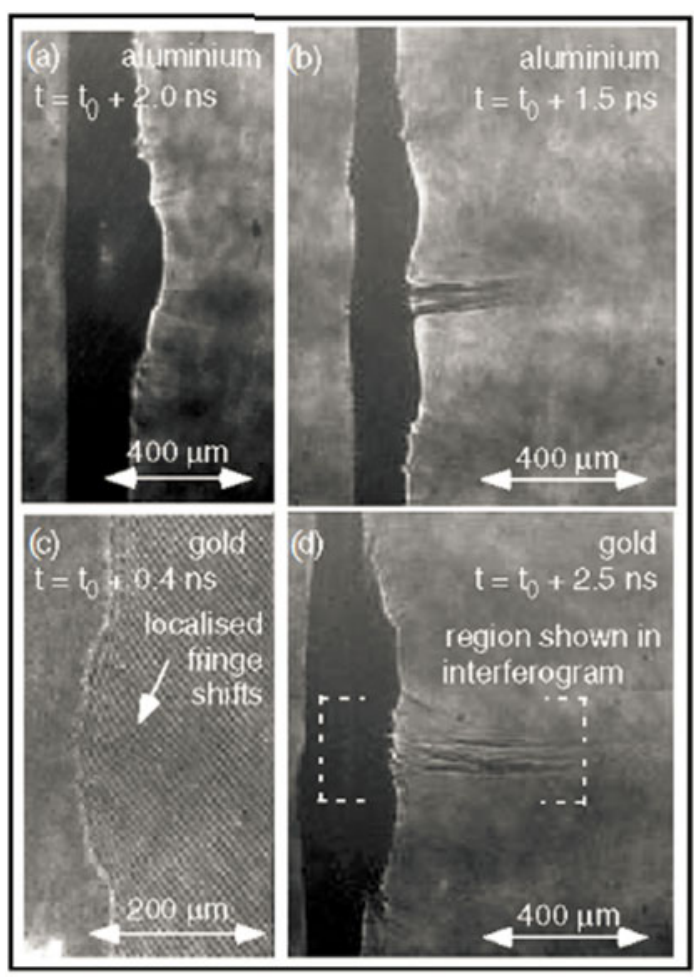

Figure 12. Probe beam images of (a), (b) aluminum targets and (c), (d) gold targets ${ }^{[50]}$.

\subsection{Laser-driven high- $\beta M R$}

In the past several decades, plenty of LDMR experiments with high $\beta$ value were performed ${ }^{[13,50,51]}$. In these experiments long-pulse (ns) lasers are employed to ablate a solid target, producing two plasma bubbles with self-generated magnetic fields, which is due to the Biermann battery effect. Two bubbles are moving toward each other due to the high pressure. Magnetic reconnection occurs when two plasma bubbles meet, and a neutral current sheet is created with the diffusion of the magnetic fields.

\subsubsection{Experiment on the Vulcan laser facility}

Nilson and Williagale ${ }^{[50]}$ performed a magnetic reconnection experiment with two laser beams interacting with a solid target in the Vulcan laser at the Rutherford Appleton Laboratory, UK. Two laser beams with a wavelength of $1.054 \mu \mathrm{m}$, a pulse of $1 \mathrm{~ns}$, and a laser intensity of $10^{15} \mathrm{~W} \cdot \mathrm{cm}^{-2}$ were ablating either an aluminum or gold foil. By changing the distance between the two laser spots, from $200 \mu \mathrm{m}$ to $400 \mu \mathrm{m}$, the dominance of thermal pressure, the magnetic pressure, and the collisionality of the interaction at the midplane were alternated. When increasing the distance between the two laser spots, the $\nabla T_{e} \times \nabla n_{e}$ self-generated magnetic field dominates the plasma dynamics in a low- $\beta$ plasma, and results in two distinct jets coming from the midplane, as shown in Figures 12(b) and 12(d), and the contractive

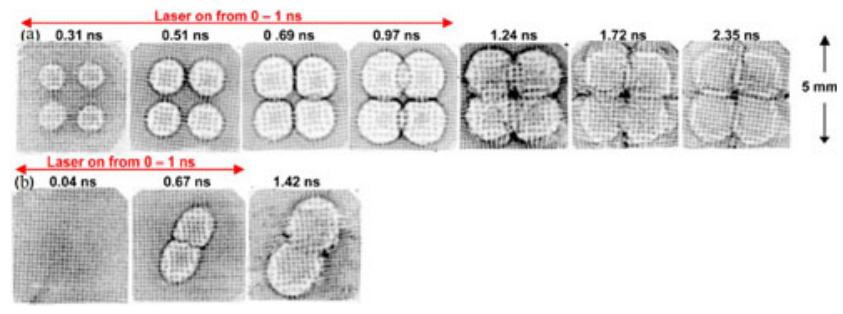

Figure 13. Proton radiography data, (a) four or (b) two laser beams were employed to ablate a $\mathrm{CH}$ foil ${ }^{[13]}$.

results were obtained with decreasing the distance between the two laser spots, as shown in Figures 12(a) and 12(c). The Thomson spectrum gives the temperature in the interaction area. All the experimental results indicate that magnetic reconnection happened rather than a standard hydrodynamic collision. Enlarging the distance between the two bubbles decreases the plasma $\beta$, while the magnetic fields will also decrease rapidly due to the expanding.

\subsubsection{Experiment on the OMEGA laser facility}

The quantitative field maps of the laser-generated plasma bubbles were revealed by Li et al. ${ }^{[13]}$ first. The experiment was performed at the OMEGA laser facility at the University of Rochester. Four or two interacting plasma bubbles were generated by four or two lasers with a wavelength of $0.351 \mu \mathrm{m}$, a pulse of $1 \mathrm{~ns}$, ablating a $\mathrm{CH}$ foil. Each beam has energy of $500 \mathrm{~J}$, with a spot diameter of $800 \mu \mathrm{m}$, and the laser intensity of $10^{14} \mathrm{~W} \cdot \mathrm{cm}^{-2}$. A fusion reaction in an implosion generated the proton backlight, which is pulsed, monoenergetic, and isotropic. These properties are critical for the experiment. The spatial structure and the temporal evolution of the magnetic field were obtained as shown in Figure 13. Particularly in Figure 13(b), by 1.42 ns, most fields have reconnected in the interaction region. From another single-laser-beam experiment ${ }^{[52]}$, obtaining the temperature $T_{e} \sim 1 \mathrm{keV}$, B-field $\sim 1 \mathrm{MG}$, and the plasma density $n_{e} \sim 10^{20}-10^{22} \mathrm{~cm}^{-3}$, the $\beta$ was calculated around 10 .

The experiments of MR in a high- $\beta$ plasma mentioned above are based on self-generated magnetic field (e.g., Biermann battery) ${ }^{[13,29]}$. Using embedded external magnetic fields in counter-propagating high energy density plasma to study MR has been performed by Fiksel on the Omega Laser System for the first time ${ }^{[53]}$. Two $1.8 \mathrm{~kJ}$ laser beams with a pulse of $2 \mathrm{~ns}$, a wavelength of $0.351 \mu \mathrm{s}$, and laser intensities of $5 \times 10^{13} \mathrm{~W} / \mathrm{cm}^{2}$ were employed to ablate two plastics $(\mathrm{CH})$, which were placed oppositely with a separating distance of $4.25 \mathrm{~mm}$. Two counter-propagating plasma plumes were generated, imposing an external magnetic field by current-carrying conductors, which were placed behind each target. When the plasma plumes were colliding and merging, stagnation of the counter-propagating magnetized ribbons was observed, as well as the reconnection layer. The third target supplied a background plasma, which allowed the 
(a)

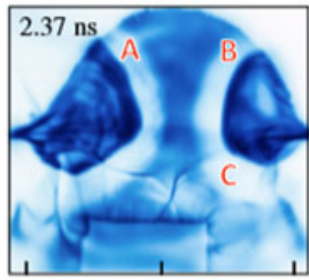

(b)

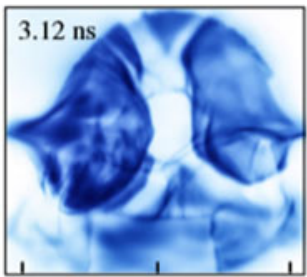

(c)

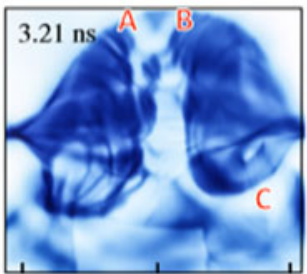

(d)

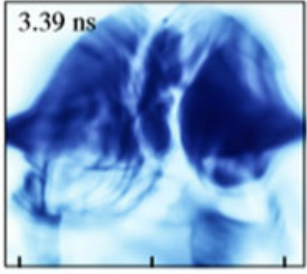

(e)

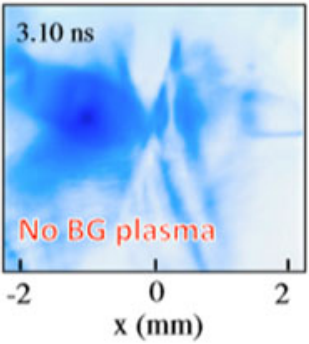

(f)

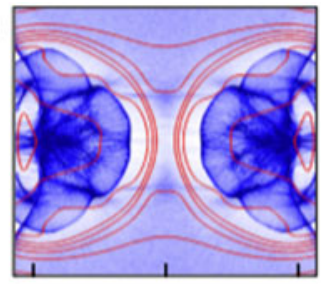

(g)

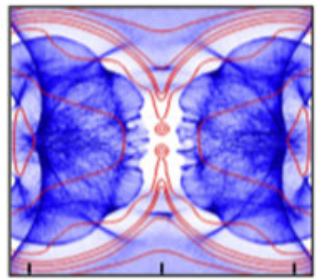

(h)

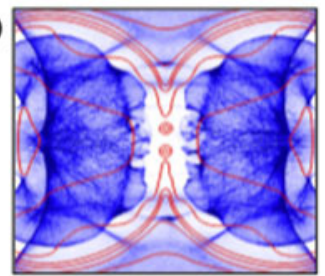

(i)

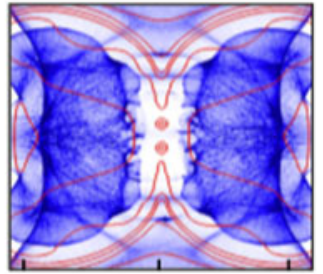

(j)

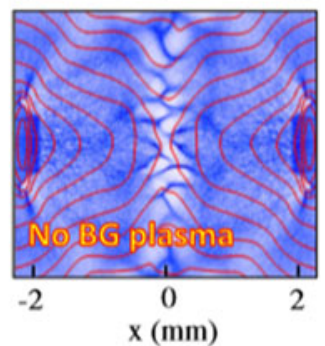

Figure 14. (a)-(e) Proton radiographic images of the magnetic field evolution. (f)-(j) Results of simulated proton radiography at the corresponding times, with overlaid magnetic field lines ${ }^{[53]}$.

magnetic field to embed into the plasma beforehand, and to be compressed by the driven plasma. Experiments without a background plasma did not show reconnection. The magnetic field at the midplane was $0 \mathrm{~T}$, and increased to $8 \mathrm{~T}$ at the targets. Figures 14(a)-14(e) show the evolution of the magnetic field. Magnetic 'ribbons' formed at $t=2.37 \mathrm{~ns}$, collided at $t=3.12 \mathrm{~ns}$, and then MR occurred, being invoked by the magnetic ribbon element connection. At $t=3.21 \mathrm{~ns}$, A and B connected, B and $\mathrm{C}$ disconnected, which was reverse at $t=2.37 \mathrm{~ns}$. And magnetic field annihilated at $t=3.39$ ns. Figures 14(f) $-14(\mathrm{j})$ show the PIC simulation results in accordance with the experimental results. The simulated reconnection rates are close to $100 \%$ of the local Alfvénic rate $B_{*} V_{A *}$. They find
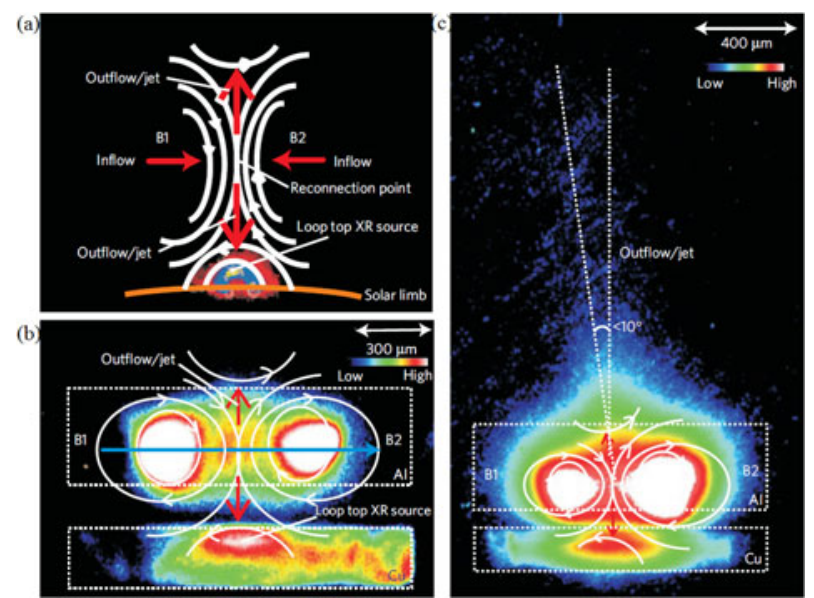

Figure 15. (a) Schematic diagram of magnetic field distribution and reconnection of the loop-top X-ray source. (b), (c) X-ray images taken by the pinhole camera in front of the target.

that the supersonic inflows cause the high compressibility in the current, which drives this extremely high reconnection rate.

\subsubsection{Experiment on the Shenguang II laser facility}

Zhong et al. ${ }^{[51]}$ performed an experiment modeling looptop X-ray source and reconnection outflows in solar flares in Shenguang II (SG II) laser facility. Four bunches of laser with long pulse $\sim 1 \mathrm{~ns}$, wavelength $0.351 \mu \mathrm{m}$, giving an incident intensity of $5 \times 10^{15} \mathrm{~W} \cdot \mathrm{cm}^{-2}$, ablated both sides of the $\mathrm{Al}$ foil target. Two plasma bubbles were produced and expanded toward each other with two toroidal MG magnetic fields due to the Biermann battery effect. When two plasma bubbles with the oppositely directed fields B1 and B2 in Figure 15(a) encountered each other, MR occurred. An arc shaped X-ray spot was found at the center of the $\mathrm{Cu}$ target, which was preset under the $\mathrm{Al}$ target, demonstrating that the plasma generated on the $\mathrm{Cu}$ target was impacted by a highspeed outflow on the $\mathrm{Al}$ foil. The picture of the arc shape of the spot resembles the loop-top X-ray source observed in solar flares. Figures $15(\mathrm{~b})$ and 15(c) show the pinhole $\mathrm{X}$-ray images, with symmetry and asymmetry laser intensities, respectively. The process was described by MHD both in laser plasmas and solar flares. According to the key plasma parameters, $T=1 \mathrm{keV}, B=100 \mathrm{~T}$, and plasma density $n_{e}=10^{19} \mathrm{~cm}^{-3}$, they obtained $\beta$ around 4 . This is the first time that the loop-top X-ray source and reconnection outflows in laboratory MR with a high- $\beta$ plasma environment were reported. More discussions are shown in Section 4.1.

Zhong et al. ${ }^{[54]}$ performed another magnetic reconnection experiment comparing the two cases, without or with guide magnetic field (a component of a magnetic field along the reconnection-induced electric field) in Shenguang II (SG II) laser facility. The experimental setup is shown in Figure 16(a). During the experiment, the orientations of the 
(a)
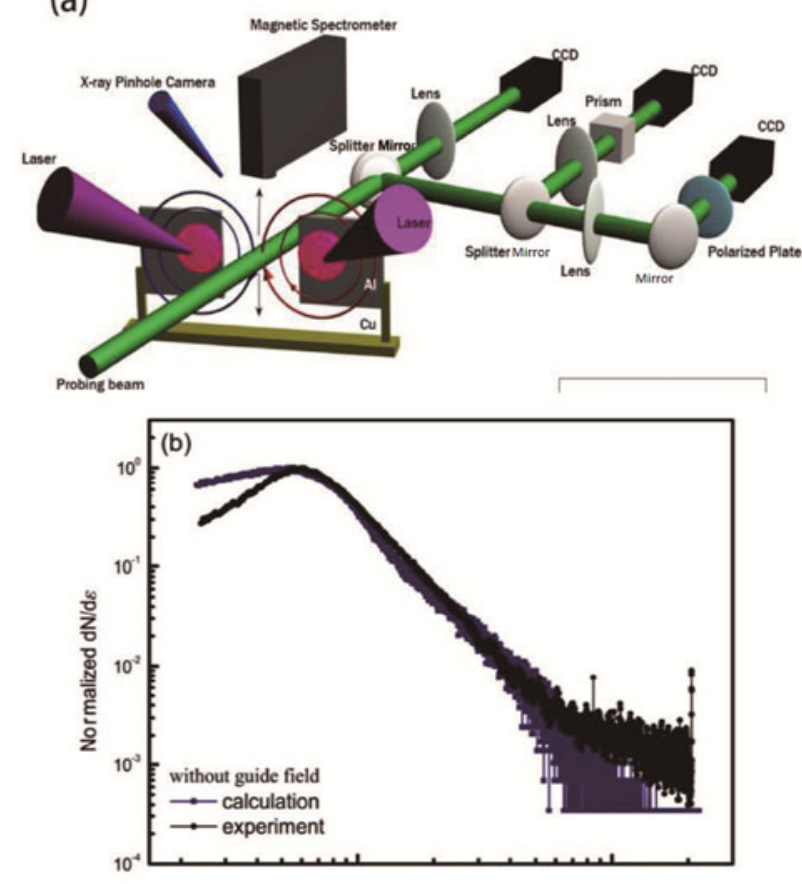

Figure 16. (a) Experimental setup of Zhong et al. (2016) ${ }^{[54]}$. (b) Black: experimental electron spectrum. Blue: simulated electron spectrum.

target and the laser beams were set in an appropriate way to achieve the two cases without or with the guide magnetic field. Figure 17(a) shows a V shape ejecting above the dark area, instead of an X shape in Figure 17(c), which is because the high plasma density blocks the probe beam. Combining Figure 17(a) and Figure 17(c), they proved that the electron is the only species accelerated by the MR in the experiment. Figure 17(b) and Figure 17(d) show the experimental and theoretical results with the guide field. In these two results, only one ejecting was found, which is because the guide field helps accelerated particles obtain more energy changing the trajectory of them ${ }^{[55]}$. They also found that with the existence of the guide field, the acceleration of non-thermal electrons is more efficient, than without a guide field, which will be discussed in Section 4.3.

\section{Applications of laser-driven magnetic reconnection}

\subsection{Modeling loop-top X-ray source and X-ray jets in solar flares}

Coronal mass ejection (CME) is a kind of eruption in the solar atmosphere. Its burst is alone with large scale ejections of mass and magnetic flux from the solar corona into interplanetary space. The CSHKP model ${ }^{[56-59]}$ is regarded as a standard model of CME. As the developments of observation and MHD theory, the understandings of the origin and evolution of CME have developed a lot ${ }^{[60-63]}$.

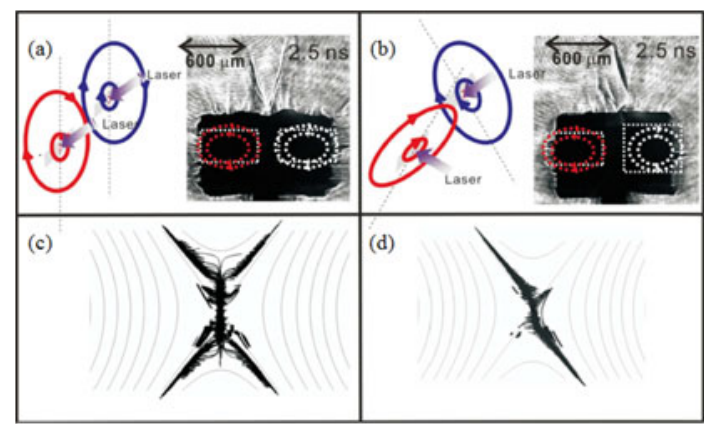

Figure 17. (a), (b) The experimental results, two solid ellipses in the left panels represent the laser-produced magnetic systems, the gray contours (shadow images) in the right panels describe the trajectories of the energetic electrons. (c), (d) The simulations results, a group of electrons moving in the EM field without or with guide field, respectively.
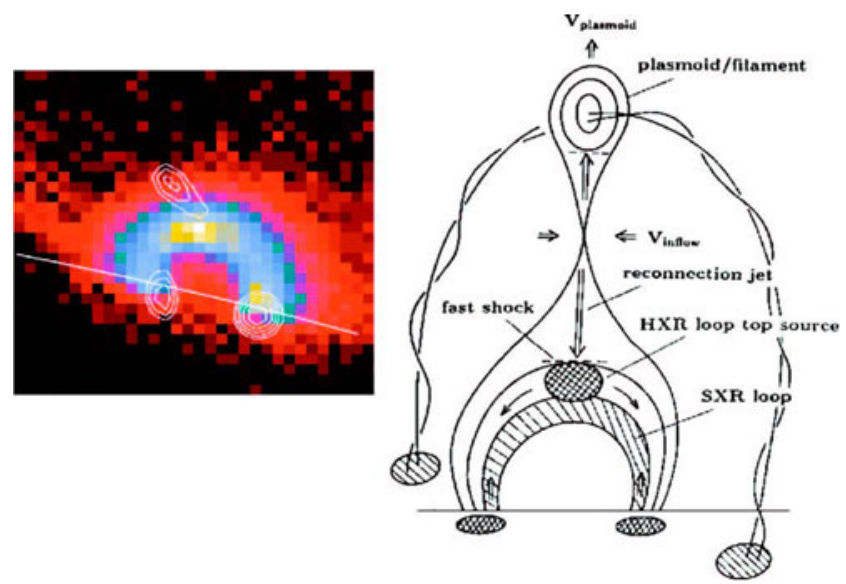

Figure 18. Hard X-ray image of a coronal arcade observed by $\mathrm{YOHKOH}$ satellite ${ }^{[2]}$, and standard model of $\mathrm{CME}^{[64]}$.

In brief, magnetic lines evolve arcade shape in the active area of corona, then antiparallel magnetic lines reconnect at an X-point. The outside mass is ejected to the space, while the particles at the reverse direction impact on the solar surface with a hot X-ray region produced. Figure 18 shows the standard model of $\mathrm{CME}^{[64]}$ and hard $\mathrm{X}$-ray image of a coronal arcade observed by YOHKOH satellite ${ }^{[65]}$.

Zhong et al. ${ }^{[51]}$ carried out an experiment to simulate the loop-top X-ray source and outflows, as we mentioned in Section 3.3.3. There were two laser spots on the $\mathrm{Al}$ foil, and their magnetic field reached $10^{6} \mathrm{G}$. Figure 15(b) shows that the $\mathrm{X}$-ray radiation of the middle region between the two spots is stronger than in the other regions, which also shows some special structures. Magnetic field lines reconnected in this region, and part of the annihilated magnetic energy was released as the X-ray emission. In outward direction, an outflow is observed, which is like a jet in the solar flare. While in the opposite direction, a $\mathrm{Cu}$ target is preset below the Al foil, a bright spot is seen (Figure 15(b)) at this position. This is the evidence that the outflow of the 
Table 1. The similarity of solar flares and the experiment of Zhong \begin{tabular}{llll} 
et al. ${ }^{[51]}$, with $a=10^{-11}, b=10^{8}$ and $c=10^{10}$ & \\
\hline Parameters & $\begin{array}{l}\text { Solar flare } \\
\text { plasma }\end{array}$ & Experiment & $\begin{array}{l}\text { Solar flare plasma } \\
\text { (scaled) }\end{array}$ \\
\hline Length $(\mathrm{cm})$ & $\sim 10^{9}-10^{10}$ & $\sim 10^{-1}$ & $\sim 10^{-2}-10^{-1}$ \\
Time $(\mathrm{s})$ & $\sim 100-1000$ & $\sim 10^{-9}$ & $\sim 10^{-9}-10^{-10}$ \\
Pressure $(\mathrm{Pa})$ & $\sim 0.001-10$ & $\sim 10^{7}$ & $\sim 10^{7}-10^{11}$ \\
Density $\left(\mathrm{cm}^{-3}\right.$ ) & $\sim 10^{9}-10^{11}$ & $\sim 10^{19}-10^{20}$ & $\sim 10^{19}-10^{21}$ \\
Velocity $\left(\mathrm{km} \cdot \mathrm{s}^{-1}\right)$ & $\sim 10-100$ & $\sim 100$ & $\sim 100-1000$ \\
Magnetic field $(\mathrm{G})$ & $\sim 10-100$ & $\sim 10^{6}$ & $\sim 10^{6}-10^{7}$
\end{tabular}

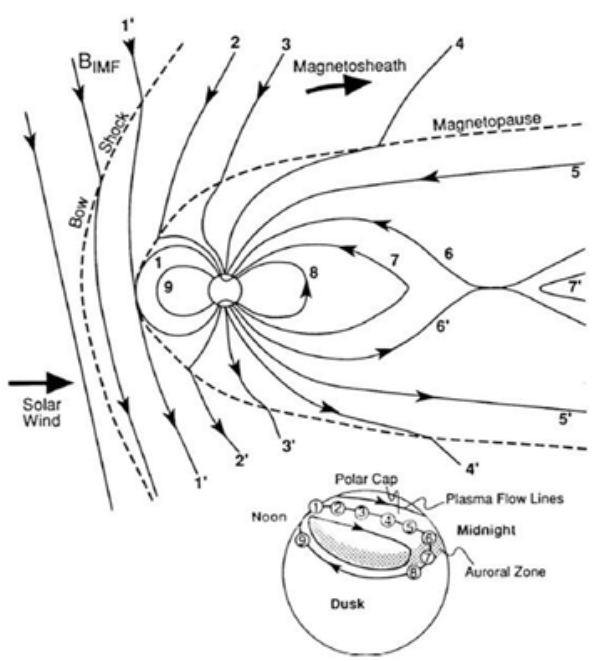

Figure 19. Schematic of the magnetic field interactions between solar wind and Earth's magnetosphere ${ }^{[69]}$.

MR impacted on the $\mathrm{Cu}$ target. The experimental magnetic field distribution and the X-ray emission agree with the observational result of the loop-top X-ray source in solar flare very well. Ryutov et al. ${ }^{[66]}$ introduced a scaling relation of two ideal MHD systems, where the Reynolds number, Peclet number and magnetic Reynolds number are very large. When the laser-produced plasmas in this experiment are scaled to the solar flare plasma, they reach a good agreement, which is shown in Table 1. This experiment is also a typical application of MR scaled to the loop-top X-ray source and outflow in solar flares.

\subsection{Solar wind and the Earth's magnetosphere}

When the solar wind reaches to the Earth's magnetosphere with a southward magnetic field, magnetic field lines of two systems may reconnect. As shown in Figure 19, MR occurs in both the dayside (magnetopause) and nightside (magnetotail) of the Earth's magnetosphere ${ }^{[67-69]}$.

Experiments have been carried out to simulate the interaction when solar wind clashes on the Earth's dipole magnetic field ${ }^{[70,71]}$. The experimental setup of Zhang et al.,
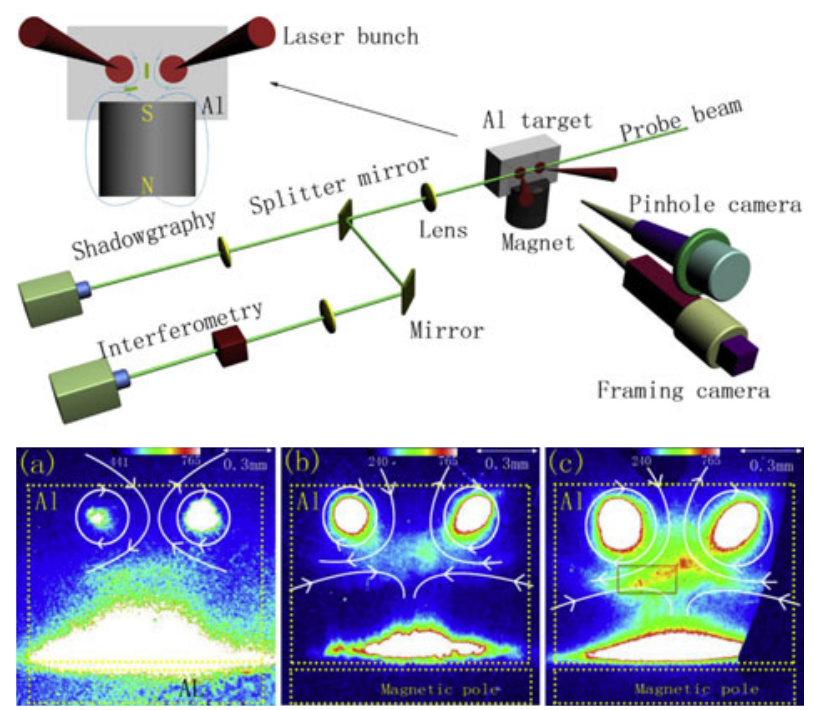

Figure 20. Upper panel: experimental setup of Zhang et al. ${ }^{[71]}$. Down panel: X-ray image of shots with magnet of different field strengths, (a) null, (b) $3000 \mathrm{G}$ and (c) $4000 \mathrm{G}$. The magnetic field is expressed as solid lines.

which was carried out at the SG II laser facility, is shown in Figure 20. The target was much like that of Zhong et al. ${ }^{[51]}$, and the obstacle was replaced by a cylindrical permanent magnet. The magnetized outflow between the two focus spots imitated the solar wind, then rushed into the magnetic field of the magnet, which was used to model the Earth's magnetosphere. According to the X-ray images, MR occurred both between fields of the plasma bubbles and field of the permanent magnet. The reconnection process became more violent as the field of magnet strengthened, where the mustache-like region appeared when the field strength changed from null to $4000 \mathrm{G}$. This experiment is believed to be helpful to understand the dynamic phenomena when solar wind interacts with the Earth's magnetosphere ${ }^{[71]}$.

The MR between the solar wind and the Earth's magnetosphere is asymmetric. The magnetic strength of the solar wind is about seven times that of the magnetopase's, and the difference of density is about a factor of 0.3 . The plasma $\beta=P_{\text {thermal }} / P_{\text {magnetic }}$ is about 0.1 in the solar wind, while about 1 in the magnetopause, and the plasma ram pressure $\beta=P_{\text {ram }} / P_{\text {magnetic }}$ on the solar wind side is 50 , so that the reconnection process is strongly driven ${ }^{[72-74]}$. In the experiment of Rosenberg et al. ${ }^{[14]}$, which was conducted at the OMEGA facility, the asymmetric effect on MR was investigated. Two reconnecting asymmetric plasma bubbles were produced, and the reconnection rate was deduced by the proton radiography. By comparing with the result of symmetric result, it is found that the reconnection rate is controlled by the relative velocities of the two bubbles flowing into the reconnection region, but not the asymmetry. In addition, the reconnection rate is slightly impacted by the out-of-plane magnetic field, which is caused by the 3D 

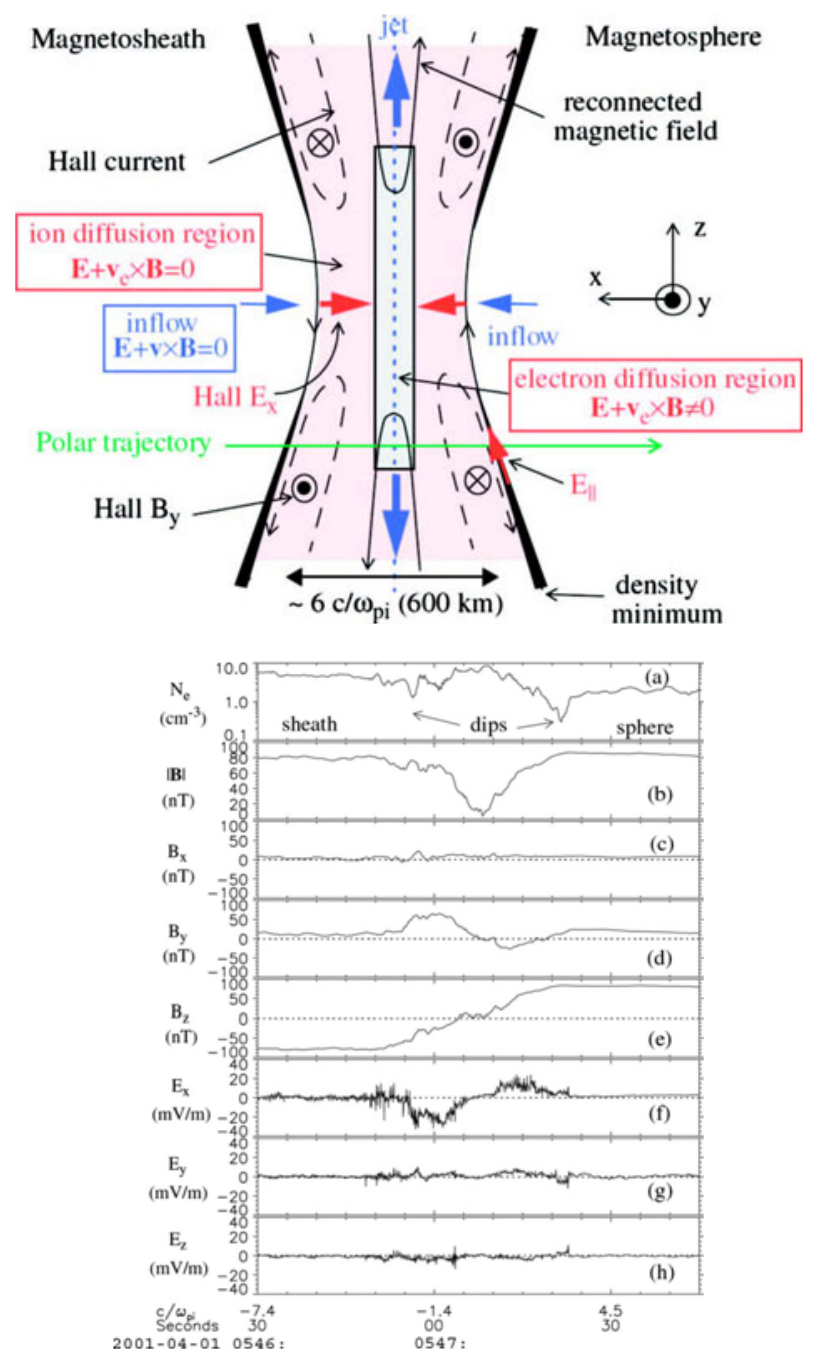

Figure 21. Upper panel: POLAR satellite trajectory through the reconnection region in the Earth's magnetosphere. Lower panel: the detail observed data ${ }^{[73]}$.

plasma geometry. However, 2D MHD simulations show that the asymmetries of magnetic field, density, resistivity and some other parameters do strongly impact the reconnection rate ${ }^{[75,76]}$. These include when the upstream magnetic ratio is 0.5 , the reconnection rate is almost one half of that of the symmetric case $\mathrm{e}^{[75]}$.

When magnetic field lines in the solar wind reconnect with the Earth's magnetosphere, the ion diffusion region has a scale size of $c / \omega_{p i} \sim 100 \mathrm{~km}^{[73]}$, which is of the order of the ion gyroradius. The plasma is in collisionless regime. In this situation, ions and electrons move separately, and two-fluid effects appear ${ }^{[77,78]}$. The upper panel of Figure 21 shows the two-fluid dynamic in the reconnection layer. As the particles move into the reconnection layer, ions become demagnetized and turn $90^{\circ}$, leaving the reconnection layer through outflow. However, electrons are still magnetized, and they flow inward along the branches of the X-region. The separating movement generates net
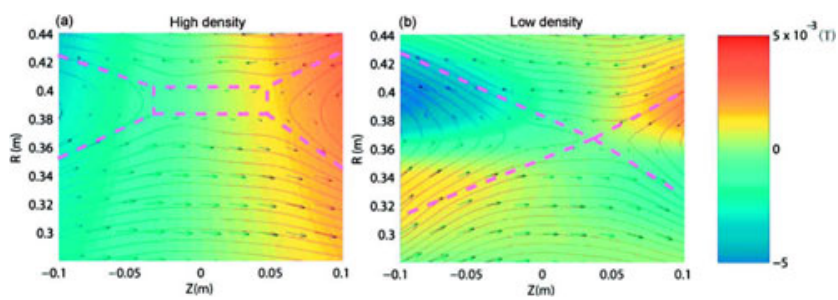

Figure 22. Measured magnetic field in the reconnection layer for (a) high density and (b) low density cases ${ }^{[4]}$.

circular currents, resulting in quadrupole magnetic field vertical to the reconnection layer ${ }^{[25]}$. In 2002 , the POLAR satellite passed through the reconnection region in the Earth's magnetosphere, and the conjecture flight path is shown in the upper panel of Figure $21^{[73]}$. The lower panel of Figure 21 shows the measured electron density and electromagnetic field in that region, and the quadrupole magnetic field structure is observed ${ }^{[73]}$. Yamada et al. ${ }^{[4,79]}$ carried out a series of experiments in the MRX to measure the magnetic field distribution of MR in the collisional regime and collisionless regime. Figure 22(a) shows the measured magnetic field of the high density case, where the ion mean free path is less than the current sheath thickness. The plasma is in collisional regime, and the magnetic field is in rectangular shape. However, in the low density case, the plasma is in collisionless regime, and two-fluid effect becomes remarkable. The magnetic field of this case roughly has quadrupole shape. This experiment confirms the twofluid effect in both theory and observation.

\subsection{Particle acceleration}

Magnetic reconnection converts magnetic energy into plasma energy, driving outflows, heating the plasma and accelerating energetic particles. When particles go deep into the reconnection region, they are unmagnetized and freely accelerated by the reconnection electric field ${ }^{[80,81]}$. There are some acceleration mechanisms, such as acceleration at magnetic islands ${ }^{[82]}$, diffusive acceleration at a fast mode shock $^{[83]}$ and second Fermi acceleration by turbulence ${ }^{[84]}$. Particles can even be accelerated to a relativistic regime. Yamada et al. ${ }^{[85]}$ reported that in the experiment on MRX device, as much as $15 \%$ of magnetic energy was converted to electron energy. Magnetic reconnection is a promising candidate for explaining energetic particles occurring in solar flares, gamma-ray burst and jets from active galactic nucleus $(\mathrm{AGNs})^{[80,86]}$.

Laser-driven magnetic reconnection is also an effective method to interpret the particle acceleration problem in the reconnection region. In the experiment of OMEGA $\mathrm{EP}^{[41]}$, the electron energetic spectra were measured by a 5channel electron spectrometer at the target rear. The spectra are shown in Figure 23(a). With adjustment of pulse-topulse delay time, MR is expected in the right panel where 
(a) Electron Measurements
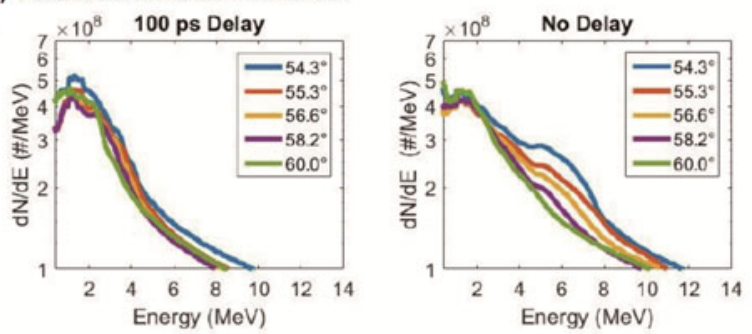

(b) Electrons accelerated into target, developing power-law spectrum.

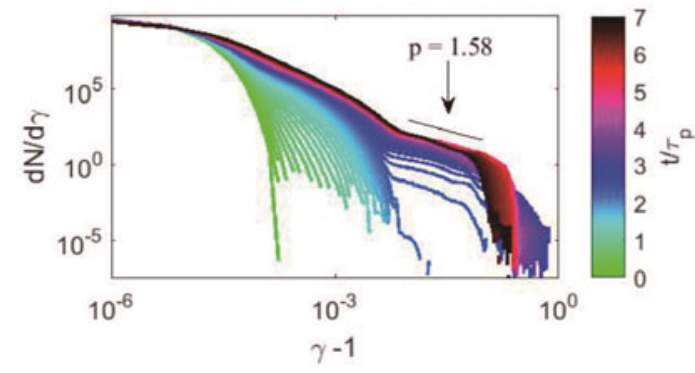

(c) Magnetic energy dissipates.

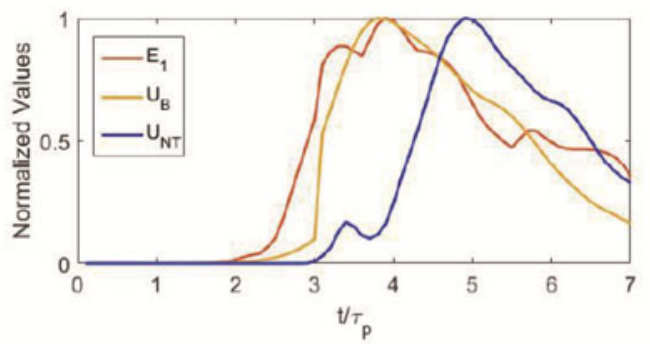

Figure 23. (a) The experimental electron spectra of OMEGA EP ${ }^{[41]}$, which were measured at the target rear by a 5-channel electron spectrometer. The left panel is shot with 100 ps pulse-to-pulse delay, and the right panel is shot with no pulse-to-pulse delay. The lines with different colors are measured with angles with respect to the rear target normal. (b) and (c) are PIC simulation results using code OSIRIS. (b) The theoretical temporal evolution of electron spectrum in the midplane region. (c) The temporal evolution of maximum reconnection electric field $\left(E_{1}\right)$, nonthermal electron energy $\left(U_{N T}\right)$ and magnetic potential energy $\left(U_{B}\right)$.

two lasers are focused on the target simultaneously, while there is no reconnection in the left panel where two lasers delay 100 ps each other. It is obvious that the non-thermal portion of the reconnection case was strengthened, which supported the association between electron acceleration and MR. Figure 23(b) shows the temporal evolution of electron spectrum in the midplane region simulated by PIC code OSIRIS. Addition to the Maxwellian low-energy portion, electrons are accelerated to a non-thermal portion, which can be fitted by a power law. Figure 23(c) shows the process that magnetic energy and electric energy convert to energy of non-thermal electrons, demonstrating the rapid acceleration by magnetic energy dissipation in the reconnection region.
Experiment by Zhong et al. ${ }^{[54]}$ on SG II provided the spectrum of energetic electron from the reconnection region. The experimental setup is shown in Figure 16(a), where two $\mathrm{Al}$ foils were driven by laser beams to generate MR and a magnetic spectrometer (MS) was used to measure the energetic electron spectrum. The collimating hole of the MS took aim in the middle between the two foils, and the distance was about $15 \mathrm{~cm}$. This setup ensured the MS to record the energetic electrons from the outflow of the reconnection region. The experimental electron spectrum is the black line in Figure 16(b), and the spectrum consists of a low-energy thermal component (below $200 \mathrm{keV}$ ) and a high energy non-thermal component (above $200 \mathrm{keV}$ ). What is more, the non-thermal component can be even further fit as a soft component with an index of 2.5 (below $500 \mathrm{keV}$ ) and harder component with an index of 1.5. In some observed spectra of flares, hardening at high energy tails was also recorded. Zhong et al. also performed simulations, which employed electrons with initial distribution of Gaussian profile and simple reconnection-induced electric field. The electron spectrum of the reconnection outflow in the simulation was plotted. The simulation well reproduced the soft component from 200 to $500 \mathrm{keV}$ in experiment, but the origin of the harder component was an unsolved problem. According to the theoretical simulation works at that time, one candidate explanation was shock formation in the reconnection region ${ }^{[55,87,88]}$. Recently, double powerlaw-shaped electron spectrum has been reproduced in $2 \mathrm{D}$ simulation using Zwingmann model ${ }^{[89]}$. The particles that are trapped and circulate around magnetic islands are the reason of the soft component of the power-law spectrum, while the escaped and partly trapped particles produce the harder component ${ }^{[00]}$. There is still a difference between the experimental and theoretical results, where the harder component has smaller power-law index than the soft component in the experiment, while the relationship is reverse in the simulation.

The theoretical electron spectrum and temporal evolution of energy conversion in simulations support that the energetic electrons in the experiment are accelerated in the reconnection region, but it still needs further experimental and theoretical works to deepen the understanding of the particle acceleration mechanism in the magnetic reconnection region. For example, experiments include the bubbles' interactions with parallel magnetic fields, which can be realized by two counter directional laser irradiations, and reconnection experiment in low- $\beta$ plasmas where it can largely reduce the thermal effects of collisions.

\subsection{Acceleration and pickup ring of energetic electrons observed in relativistic magnetic reconnection}

The origins of the non-thermal radiation of gamma-ray bursts and jets of AGNs are outstanding issues of astrophysics ${ }^{[91,92]}$. It has been proposed that such non-thermal 

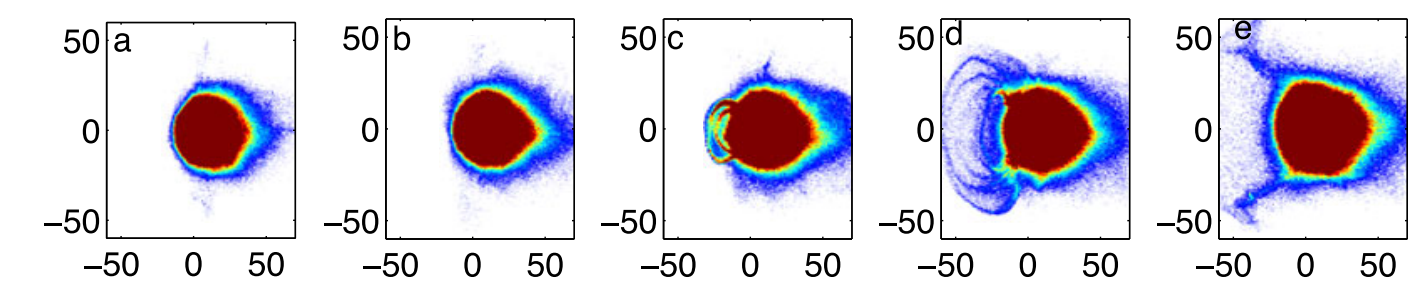

A
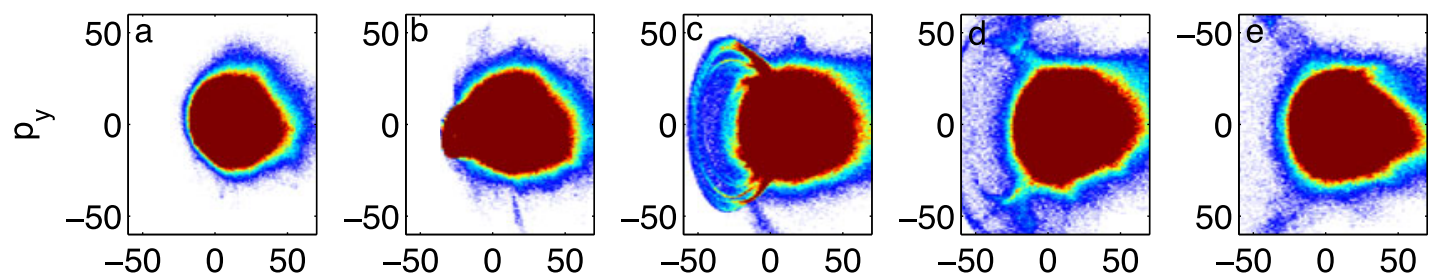

B
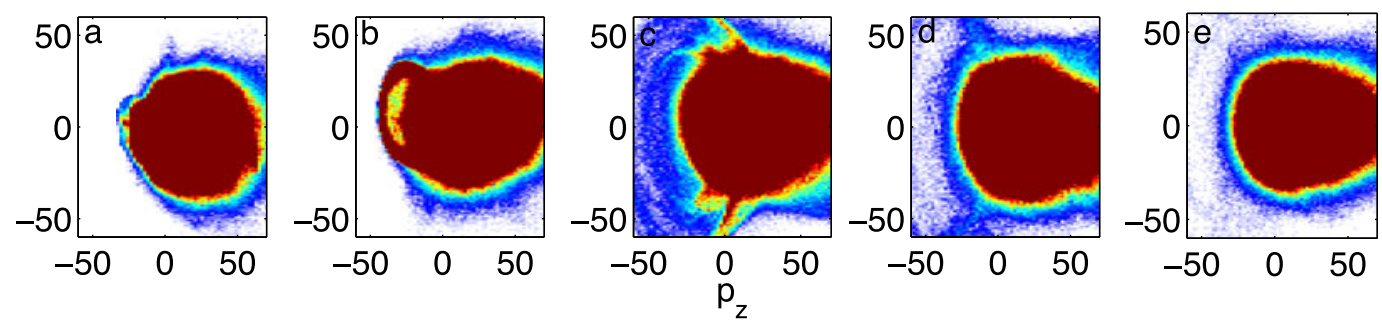

Figure 24. The electron distributions in the phase space of $\left(p_{z}, p_{y}\right)$. From the left to right, the columns correspond to the time $t=40 T_{0}, 45 T_{0}, 50 T_{0}, 55 T_{0}$ and $60 T_{0}$, respectively. Row A is for $I_{20}=3 \times 10^{20} \mathrm{~W} / \mathrm{cm}^{2}$, row B is for $I_{20}=5 \times 10^{20} \mathrm{~W} / \mathrm{cm}^{2}$ and row C is for $I_{20}=7 \times 10^{20} \mathrm{~W} / \mathrm{cm}^{2[97]}$.

radiation could be produced from ultrarelativistic jet outflows picking up ambient interstellar protons and electrons $^{[93-96]}$.

The electron acceleration in MR process occurring in intense femtosecond laser pulse interaction with plasmas is studied ${ }^{[97]}$. According to the analysis of the electrons located in the reconnection current sheet, the 'pickup ring' is observed. Figure 24 shows the results of the experiments with various laser intensities, such as $I_{20}=3 \times 10^{20} \mathrm{~W} / \mathrm{cm}^{2}$ for row $\mathrm{A}, I_{20}=5 \times 10^{20} \mathrm{~W} / \mathrm{cm}^{2}$ for row $\mathrm{B}$, and $I_{20}=$ $7 \times 10^{20} \mathrm{~W} / \mathrm{cm}^{2}$ for row C. Specifically, the phase space distributions of electrons of $\left(p_{z}, p_{y}\right)$ in the reconnection region at more time steps are shown in Figure 24(B) with the same simulation conditions used by Ping et al. ${ }^{[43]}$. The acceleration along the negative $z$-direction is clearly due to the reconnection electric field $E_{z}$ (in $\left(p_{z}, p_{y}\right)$ ). A novel structure in the phase space of $\left(p_{z}, p_{y}\right)$ is shown in Figure $24 \mathrm{~B}(\mathrm{c})$. This structure is very similar to the pickup ring (though distorted by relativistic effects) of solar wind protons ${ }^{[98]}$. This shows that a large portion of lowenergy electrons are 'picked up' by a frame moving in the negative $z$-direction with a velocity of $v_{z} \approx-0.9992 c\left(p_{z} \approx\right.$ $-25)$, corresponding to the Alfvén velocity of the electron, $V_{A e} \sim c / \sqrt{\epsilon}$, where $\epsilon=1+2\left(\omega_{p e 0} / \Omega_{c}\right)^{2}$ is the relative permittivity with the electron cyclotron frequency $\Omega_{c}=$ $e B / m_{e} c$ and $\omega_{p e 0}=\left(4 \pi e^{2} n_{0} / m_{e}\right)^{-1 / 2}$. All these features appearing in the MR process become weaker at a later time of $t=60 T_{0}$ (Figure 24B(e)) when the most intensive MR process is over. Particularly the 'pickup ring' (an ellipse in the relativistic regime) is diffused in both momentum and coordinate spaces, as seen in Figures 24B(c)-24B(e). This suggests a quasilinear diffusion mechanism of wave particle resonance.

Then, similar simulations are performed with different laser peak intensities. Figure 24(A) presents the phase space distributions of electrons of $\left(p_{z}, p_{y}\right)$ and $\left(p_{z}, p_{x}\right)$ for the lasers with a peak intensity $I_{0}=3 \times 10^{20} \mathrm{~W} / \mathrm{cm}^{2}$. The 'pickup ring' is again observed in Figures 24A(c)24A(e). Comparing with Figure 24(B), the 'pickup ring' has already occurred for the lasers with the stronger $I_{20}=$ $5 \times 10^{20} \mathrm{~W} / \mathrm{cm}^{2}$, but it does not occur for the lasers with the weaker $I_{10}=3 \times 10^{20} \mathrm{~W} / \mathrm{cm}^{2}$, until at $t=50 T_{0}$.

Figure 24(C) presents the phase space distributions of electrons of $\left(p_{z}, p_{y}\right)$ and $\left(p_{z}, p_{x}\right)$ for the lasers with $I_{30}=7 \times 10^{20} \mathrm{~W} / \mathrm{cm}^{2}$. The 'pickup ring' occurs at $t=$ $45 T_{0}$ in Figure $24 \mathrm{C}(\mathrm{b})$. By comparing Figure $24(\mathrm{C})$ with Figure 24(A), the 'pickup ring' is larger at the same time, indicating that the electrons are accelerated to higher energy for higher laser intensity. The evolution of the pickup ring of energetic electrons shown at different laser peak intensities suggests that it is produced during the magnetic reconnection process rather than due to some instability.

Figure 25 shows the electron energy spectra in all simulation box and reconnection current sheet. For the entire electron population, the energy spectra of the reconnection with two lasers and non-reconnection with one laser, shown in Figure 25(a), are very similar to each other, indicating 

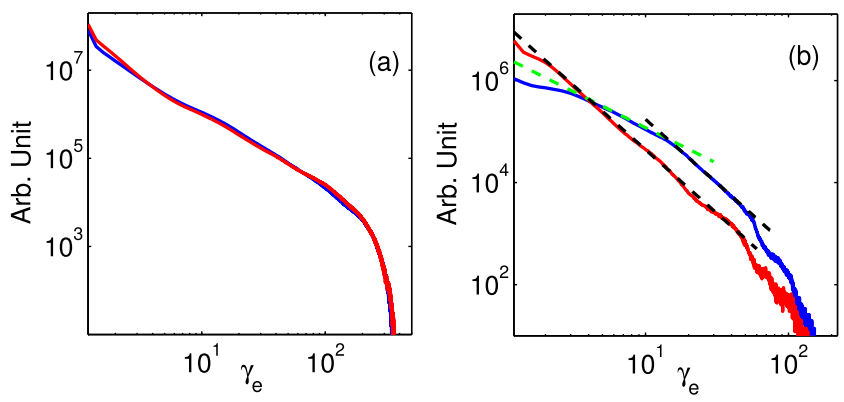

Figure 25. Energy spectra for the electrons (a) in the entire simulation box and (b) in the reconnection region only for $I_{20}=5 \times 10^{20} \mathrm{~W} / \mathrm{cm}^{2}$. The solid blue curves are for the two-laser case and the red for the one laser case. In (a) the red line has been multiplied by a factor of 2 to compare with the blue line with two lasers. In (b) the dashed lines indicate the power law of the spectrum, $\gamma_{e}^{-p}$, with the black line for $p=2.5$ and the green line for $p=1.4^{[97]}$.

that the number of electrons accelerated by MR is only a small portion compared to the electrons accelerated by the lasers. Nevertheless, the spectra in the reconnection region, plotted in Figure 25(b), demonstrate substantial acceleration of electrons by MR in the relativistic regime $\left(\gamma_{e}>4\right)$ with an energy gain of $\delta \gamma_{e} \sim 10-100$. Moreover, the spectra for the two cases show different power-law profiles $\gamma_{e}^{p}$. For non-reconnection with one laser case, the power is approximately $p=2.5$ with a knee in the highly relativistic regime $\left(\gamma_{e}>45\right)$. On the other hand, for the case with $\mathrm{MR}$, the spectrum is changed to a power of $p=1.4$ in the $\mathrm{MeV}$ relativistic regime of $4<\gamma_{e}<\gamma_{\text {pon }}$, where $\gamma_{\text {pon }} \sim a_{0}$ is the peak ponderomotive potential of the laser pulse. It recovers the power law for the single laser case with $p=2.5$ in the intermediate relativistic regime of $\gamma_{\text {pon }}<\gamma<50$, nevertheless with an energy gain of $\delta \gamma_{e} \sim 20-40$. In the higher energy regime of the knee region, the energy gain is up to $\delta \gamma_{e} \sim 100$. Thus, the spectra shown in Figure 25(b) demonstrate clearly the electron acceleration by MR in the relativistic regime. Note that the power law of $p=1.4$ below $\gamma_{e}=\gamma_{\text {pon }}$, is similar to the observed gamma-ray burst spectra, which have a flatter slope than that produced by the internal shocks mechanism ${ }^{[99,100]}$, though the particle energy in our simulation is still much below the GeV level of the gamma-ray bursts. Moreover, the flat electron spectra are presented solar flares and other explosive events in the corona, where the particles are accelerated in reconnecting current sheets with or without guide field ${ }^{[87,88,90]}$.

\section{Summary}

In summary, we have introduced several MR experiments in low- $\beta$ and high- $\beta$ plasma environments, respectively. Two mechanisms, magnetic driven and laser driven, can produce low- $\beta$ plasma. And using long-pulse lasers to ablate solid target is the typical way to generate high- $\beta$ plasma.
These laboratory experiments can be applied to study the fundamental magnetic reconnection physical issues, and simulate the fundamental processes in astrophysics.

\section{Acknowledgement}

This work was supported by the Science Challenge Project (No. TZ2016005), the National Basic Research Program of China (No. 2013CBA01503), the National Natural Science Foundation of China (Nos. 10905004, 11220101002, and 11622323), and the Fundamental Research Funds for the Central Universities.

\section{References}

1. M. Kivelson and C. Russell, Introduction to Space Physics (Cambridge University Press, Cambridge, 1995), p. 92.

2. S. Masuda, T. Kosugi, H. Hara, S. Tsuneta, and Y. Ogawara, Nature 371, 495 (1994).

3. E. Priest and T. Forbes, Magnetic Reconnection (Cambridge University Press, Cambridge, 2000).

4. M. Yamada, Y. Ren, H. Ji, J. Breslau, S. Gerhardt, R. Kulsrud, and A. Kuritsyn, Phys. Plasmas. 13, 052119 (2006).

5. J. Egedal, A. Fasoli, M. Porkolab, and D. Tarkowski, Rev. Sci. Instrum. 71, 3351 (2000).

6. B. E. Chapman, A. F. Almagri, J. K. Anderson, T. M. Biewer, P. K. Chattopadhyay, C.-S. Chiang, D. Craig, D. J. DenHartog, G. Fiksel, C. B. Forest, A. K. Hansen, D. Holly, N. E. Lanier, R. O'Connell, S. C. Prager, J. C. Reardon, J. S. Sarff, M. D. Wyman, D. L. Brower, W. X. Ding, Y. Jiang, S. D. Terry, P. Franz, L. Marrelli, and P. Martin, Phys. Plasmas. 9, 2061 (2002).

7. R. J. Hawryluk, Rev. Mod. Phys. 70, 537 (1998).

8. J. Johner, J. Fusion Sci. Technol. 59, 308 (2011).

9. H. Ji, M. Yamada, S. Prager, W. Daughton, and V. Roytershteyn, Bull. Am. Phys. Soc. 55, 15 NP982 (2010).

10. H. T. Ji and W. Daughton, Phys. Plasmas. 18, 111207 (2011).

11. M. A. Yates, D. B. van Hulsteyn, H. Rutkowski, G. Kyrala, and J. U. Brackbill, Phys. Rev. Lett. 49, 1702 (1982).

12. P. M. Nilson, L. Willingale, M. C. Kaluza, C. Kamperidis, S. Minardi, M. S. Wei, P. Fernandes, M. Notley, S. Bandyopadhyay, M. Sherlock, R. J. Kingham, M. Tatarakis, Z. Najmudin, W. Rozmus, R. G. Evans, M. G. Haines, A. E. Dangor, and K. Krushelnick, Phys. Rev. Lett. 97, 255001 (2006).

13. C. K. Li, F. H. Séguin, J. A. Frenje, J. R. Rygg, R. D. Petrasso, R. P. J. Town, P. A. Amendt, S. P. Hatchett, O. L. Landen, A. J. Mackinnon, P. K. Patel, M. Tabak, J. P. Knauer, T. C. Sangster, and V. A. Smalyuk, Phys. Rev. Lett. 99, 055001 (2007).

14. M. J. Rosenberg, C. K. Li, W. Fox, I. Igumenshchev, F. H. Séguin, R. P. J. Town, J. A. Frenje, C. Stoeckl, V. Glebov, and R. D. Petrasso, Nat. Commun. 6, 6190 (2015).

15. L. Biermann, Z. Naturforsch 5A, 65 (1950).

16. Y.-T. Li, J. Zhang, and W. M. Jiang, Physics 29, 211 (2000).

17. G. Gregori, A. Ravasio, C. D. Murphy, K. Schaar, A. Baird, A. R. Bell, A. Benuzzi-Mounaix, R. Bingham, C. Constantin, R. P. Drake, M. Edwards, E. T. Everson, C. D. Gregory, Y. Kuramitsu, W. Lau, J. Mithen, C. Niemann, H.-S. Park, B. A. Remington, B. Reville, A. P. L. Robinson, D. D. Ryutov, Y. Sakawa, S. Yang, N. C. Woolsey, M. Koenig, and F. Miniati, Nature 481, 480 (2012). 
18. H. Xu, B. W. O'Shea, D. C. Collins, M. L. Norman, H. Li, and S. Li, Astrophys. J. 688, L57 (2008).

19. J. A. Stamper and B. H. Ripin, Phys. Rev. Lett. 34, 138 (1975).

20. U. Wagner, M. Tatarakis, A. Gopal, F. N. Beg, E. L. Clark, A. E. Dangor, R. G. Evans, M. G. Haines, S. P. D. Mangles, P. A. Norreys, M.-S. Wei, M. Zepf, and K. Krushelnick, Phys. Rev. E 70, 026401 (2004).

21. K. F. F. Law, M. Bailly-Grandvaux, A. Morace, S. Sakata, K. Matsuo, S. Kojima, S. Lee, X. Vaisseau, Y. Arikawa, A. Yogo, K. Kondo, Z. Zhang, C. Bellei, J. J. Santos, S. Fujioka, and H. Azechi, Appl. Phys. Lett. 108, 091104 (2016).

22. S. Fujioka, Z. Zhang, K. Ishihara, K. Shigemori, Y. Hironaka, T. Johzaki, A. Sunahara, N. Yamamoto, H. Nakashima, T. Watanabe, H. Shiraga, H. Nishimura, and H. Azechi, Sci. Rep. 3, 1170 (2013).

23. G. A. Gary, Solphys 203, 71 (2001).

24. M. Yamada, H. Ji, S. Hsu, T. Carter, R. Kulsrud, Y. Ono, and F. Perkins, Phys. Rev. Lett. 78, 3117 (1997).

25. M. Yamada, R. Kulsrud, and H. Ji, Rev. Mod. Phys. 82, 603 (2010).

26. E. G. Zweibel and M. Yamada, Annu. Rev. Astron. Astrophys. 47, 291 (2009).

27. H. Ji, M. Yamada, S. Hsu, and R. Kulsrud, Phys. Rev. Lett. 80, 3256 (1998).

28. J. Egedal, W. Fox, N. Katz, M. Porkolab, K. Reim, and E. Zhang, Phys. Rev. Lett. 98, 015003 (2007).

29. H. Ji and W. Daughton, Phys. Plasmas. 18, 111207 (2011).

30. H. Daido, F. Miki, K. Mima, M. Fujita, and K. Sawai, Phys. Rev. Lett. 56, 846 (1986).

31. C. Courtois, A. D. Ash, D. M. Chambers, R. A. D. Grundy, and N. C. J. Woolsey, Appl. Phys. 98, 054913 (2005).

32. S. Fujioka, Z. Zhang, K. Ishihara, K. Shigemori, Y. Hironaka, T. Johzaki, A. Sunahara, N. Yamamoto, H. Nakashima, T. Watanabe, H. Shiraga, H. Nishimura, and H. Azechi, Sci. Rep. 3, 1170 (2013).

33. J. J. Santos, M. Bailly-Grandvaux, L. Giuffrida, P. Forestier-Colleoni, S. Fujioka, Z. Zhang, P. Korneev, R. Bouillaud, S. Dorard, D. Batani, M. Chevrot, J. E. Cross, R. Crowston, J.-L. Dubois, J. Gazave, G. Gregori, E. d'Humières, S. Hulin, K. Ishihara, S. Kojima, E. Loyez, J.-R. Marquès, A. Morace, P. Nicolaï, O. Peyrusse, A. Poyé, D. Raffestin, J. Ribolzi, M. Roth, G. Schaumann, F. Serres, V. T. Tikhonchuk, P. Vacar, and N. Woolsey, arXiv: 1503.00247 (2015)

34. L. Gao, H. Ji, G. Fiksel, W. Fox, M. Evans, and N. Alfonso, Phys. Plasmas. 23, 043106 (2016).

35. X. X. Pei, J. Y. Zhong, Y. Sakawa, Z. Zhang, K. Zhang, H. G. Wei, Y. T. Li, Y. F. Li, B. J. Zhu, T. Sano, Y. Hara, S. Kondo, S. Fujioka, G. Y. Liang, F. L. Wang, and G. Zhao, Phys. Plasmas. 23, 032125 (2016).

36. P. Elleaume, O. Chubar, and J. Chavanne, Proc. IEEE 3, 3509 (1997).

37. M. Hoshino and Y. Lyubarsky, Space Sci. Rev. 173, 521 (2012).

38. E. D. Feigelson and T. Montmerle, Annu. Rev. Astron. Astrophys. 37, 363 (1999).

39. C. Thompson, Mon. Not. R. Astron. Soc. 270, 480 (1994).

40. B. F. Liu, S. Mineshige, and K. Ohsuga, Astrophys. J. 587, 571 (2003).

41. A. Raymond, C. F. Dong, A. McKelvey, C. Zulick, N. Alexander, T. Batson, A. Bhattacharjee, P. Campbell, H. Chen, V. Chvykov, E. Del Rio, P. Fitzsimmons, W. Fox, B. Hou, A. Maksimchuk, C. Mileham, J. Nees, P. M. Nilson,
C. Stoeckl, A. G. R. Thomas, M. S. Wei, V. Yanovsky, L. Willingale, and K. Krushelnick, arXiv:1610.06866 (2016).

42. J. A. Stamper, Laser Particle Beams 9, 841 (1991).

43. Y. Ping, J. Zhong, X. Wang, B. Liu, Y. Li, X. Yan, X. He, J. Zhang, and G. Zhao, Phys. Rev. E. 89, 031101(R) (2014).

44. J. Nycander and M. B. Isichenko, Phys. Fluids B 2, 2042 (1990).

45. S. K. Yadav, A. Das, and P. Kaw, Phys. Plasmas 15, 062308 (2008).

46. M. Yamada, Phys. Plasmas 14, 058102 (2007).

47. S. V. Bulanov, F. Pegoraro, and A. S. Sakharov, Phys. Fluids B. 4, 2499 (1992).

48. H. Che, J. F. Drake, and M. Swisdak, Nature 474, 184 (2011).

49. K. Fujimoto and D. S. Richard, Phys. Rev. Lett. 109, 265004 (2012).

50. P. M. Nilson, L. Willingale, M. C. Kaluza, C. Kamperidis, S. Minardi, M. S. Wei, P. Fernandes, M. Notley, S. Bandyopadhyay, M. Sherlock, R. J. Kingham, M. Tatarakis, Z. Najmudin, W. Rozmus, R. G. Evans, M. G. Haines, A. E. Dangor, and K. Krushelnick, Phys. Rev. Lett. 97, 255001 (2006).

51. J. Zhong, Y. Li, X. Wang, J. Wang, Q. Dong, C. Xiao, S. Wang, X. Liu, L. Zhang, L. An, F. Wang, J. Zhu, Y. Gu, X. He, G. Zhao, and J. Zhang, Nat. Phys. 6, 984 (2010).

52. C. K. Li, F. H. Séguin, J. A. Frenje, J. R. Rygg, R. D. Petrasso, R. P. J. Town, P. A. Amendt, S. P. Hatchett, O. L. Landen, A. J. Mackinnon, P. K. Patel, V. A. Smalyuk, T. C. Sangster, and J. P. Knauer, Phys. Rev. Lett. 97, 135003 (2006).

53. G. Fiksel, W. Fox, A. Bhattacharjee, D. H. Barnak, P.-Y. Chang, and K. Germaschewski, Phys. Rev. Lett. 113, 105003 (2014).

54. J. Y. Zhong, J. Lin, Y. T. Li, K. Zhang, D. W. Yuan, Y. L. Ping, H. G. Wei, J. Q. Wang, L. N. Su, F. Li, B. Han, G. Q. Liao, C. L. Yin, Y. Fang, X. Yuan, C. Wang, J. R. Sun, G. Y. Liang, F. L. Wang, Y. K. Ding, X. T. He, J. Q. Zhu, Z. M. Sheng, G. Li, G. Zhao, and J. Zhang, Astrophys. J. Suppl. 225, 30 (2016).

55. Y. Li and J. Lin, Sol. Phys. 279, 91 (2012).

56. H. Carmichael, NASA Special Publication (1964), Vol. 50, p. 451.

57. P. A. Sturrock, Nature 211, 695 (1966).

58. T. Hirayama, Sol. Phys. 34, 323 (1974).

59. R. A. Kopp and G. W. Pneuman, Sol. Phys. 50, 85 (1976).

60. J. Lin and T. G. Forbes, J. Geophys. Res. 105, 2375 (2000).

61. J. Lin, W. Soon, and S. L. Baliunas, New Astron. Rev. 47, 53 (2003).

62. T. G. Forbes, J. A. Linker, J. Chen, C. Cid, J. Kóta, M. A. Lee, G. Mann, Z. Mikić, M. S. Potgieter, J. M. Schmidt, G. L. Siscoe, R. Vainio, S. K. Antiochos, and P. Riley, Space Sci. Rev. 123, 251 (2006).

63. B. Schmieder, P. Démoulin, and G. Aulanier, Adv. Space Res. 51, 1967 (2013).

64. K. Shibata, S. Masuda, M. Shimojo, H. Hara, T. Yokoyama, S. Tsuneta, T. Kosugi, and Y. Ogawara, Astrophys. J. Lett. 451, L83 (1995).

65. S. Masuda, T. Kosugi, H. Hara, S. Tsuneta, and Y. Ogawara, Nature 371, 495 (1994).

66. D. D. Ryutov, R. P. Drake, and B. A. Reminton, Astrophys. J. Suppl. Ser. 127 (2000).

67. V. M. Vasyliunas, Rev. Geophys. 13, 303 (1975).

68. J. W. Dungey, Phys. Magnetopause 90, 17 (1995).

69. M. G. Kivelson and C. T. Russell, Science 269, 862 (1995).

70. P. Brady, T. Ditmire, W. Horton, M. L. Mays, and Y. Zakharov, Phys. Plasmas. 16, 043112 (2009). 
71. K. Zhang, J. Y. Zhong, J. Q. Wang, X. X. Pei, H. G. Wei, D. W. Yuan, Z. W. Yang, C. Wang, F. Li, B. Han, C. L. Yin, G. Q. Liao, Y. Fang, S. Yang, X. H. Yuan, Y. Sakawa, T. Morita, Z. R. Cao, S. E. Jiang, Y. K. Ding, Y. Kuramitsu, G. Y. Liang, F. L. Wang, Y. T. Li, J. Q. Zhu, J. Zhang, and G. Zhao, High Energy Density Phys. 17, 32 (2015).

72. T.-D. Phan, G. Paschmann, W. Baumjohann, N. Sckopke, and H. Luehr, J. Geophys. Res. 99, 121 (1994).

73. F. S. Mozer, S. D. Bale, and T. D. Phan, Phys. Rev. Lett. 89, 015002 (2002).

74. J. P. Eastwood, T. D. Phan, M. Øieroset, M. A. Shay, K. Malakit, M. Swisdak, J. F. Drake, and A. Masters, Plasma Phys. Contr. Fusion 55, 124001 (2013).

75. N. A. Murphy, M. P. Miralles, C. L. Pope, J. C. Raymond, H. D. Winter, K. K. Reeves, D. B. Seaton, A. A. van Ballegooijen, and J. Lin, Astrophys. J. 751, 56 (2012).

76. N. A. Murphy, A. K. Young, C. Shen, J. Lin, and L. Ni, Phys. Plasmas 20, 061211 (2013).

77. P. L. Pritchett, J. Geophys. Res. 106, 25961 (2001).

78. J. Birn and E. R. Priest (eds), Reconnection of Magnetic Fields: Magnetohydrodynamics and Collisionless Theory and Observations (Cambridge University Press, Cambridge, 2007), p. 87.

79. M. Yamada, Phys. Plasmas 14, 058102 (2007).

80. S. R. Totorica, T. Abel, and F. Fiuza, Phys. Plasmas. 24, 041408 (2017).

81. Y. E. Litvinenko, Astrophys. J. 462, 997 (1996).

82. J. F. Drake, M. Swisdak, H. Che, and M. A. Shay, Nature 443, 553 (2006).
83. S. Tsuneta and T. Naito, Astrophys. J. Lett. 495, L67 (1998). 84. J. R. Miller and N. T. O'Neill, Geophys. Res. 102, 29 (1997).

85. M. Yamada, J. Yoo, J. Jara-Almonte, H. Ji, R. M. Kulsrud, and C. E. Myers, Nat. Commun. 5, 4774 (2014).

86. M. Hoshino and Y. Lyubarsky, Space Sci. Rev. 173, 521 (2012).

87. Y. Li, H. D. Winter, N. A. Murphy, L. Jun, and W. Ning, Publ. Astron. Soc. Jpn 65, 101 (2013).

88. G. Li, X. Kong, G. Zank, and Y. Chen, Astrophys. J. 769, 22 (2013).

89. W. Zwingmann, J. Geophys. Res. 88, 9101 (1983).

90. Y. Li, N. Wu, and J. Lin, Astron. Astrophys. 605, A120 (2017).

91. E. Waxman, Plasma Phys. Contr. Fusion 48, B137 (2006).

92. E. Nakar, Phys. Rep. 442, 166 (2007).

93. R. Schlickeiser, M. Pohl, and R. Vainio, Astrophys. J. 596, 840 (2003)

94. R. Schlickeiser, R. Vainio, M. Böttcher, I. Lerche, M. Pohl, and C. Schuster, Astron. Astrophys. 393, 69 (2002).

95. R. Schlickeiser and C. D. Dermer, Astron. Astrophys. 360, 789 (2000).

96. D. Gerbig and R. Schlickeiser, Astrophys. J. 664, 750 (2007).

97. Y. L. Ping, J. Y. Zhong, X. G. Wang, Z. M. Sheng, and G. Zhao, Astrophys. J. 849, 137 (2017).

98. C. Wu and R. Davidson, J. Geophys. Res. 77, 5399 (1972).

99. G. Ghisellini, A. Celotti, and D. Lazzati, Mon. Not. Roy. Astron. Soc. 313, L1 (2000).

100. K. Asano and T. Terasawa, Astrophys. J. 705, 1714 (2009). 